\title{
Polarimetric Decomposition Over Glacier Ice Using Long-Wavelength Airborne PolSAR
}

\author{
Jayanti J. Sharma, Student Member, IEEE, Irena Hajnsek, Senior Member, IEEE, \\ Konstantinos P. Papathanassiou, Senior Member, IEEE, and Alberto Moreira, Fellow, IEEE
}

\begin{abstract}
In recent years, there has been increased interest in using synthetic aperture radar (SAR) to study and monitor glaciers and ice sheets for glaciological and climate change research. However, due to the medium's complexity, SAR backscattering from ice remains poorly understood, including the relative importance of scattering from surface and volume layers and also dependences on frequency and glacier zone. Extreme weather conditions can result in quickly changing surface conditions influencing backscatter signatures while leaving the underlying volume of interest unchanged. Surface and volume components must thus be separated in order to infer information regarding the properties of the ice volume. This paper describes a three-component scattering model to decompose polarimetric SAR (PolSAR) images of glacier ice. Total backscatter is modeled as the incoherent summation of surface, volume, and sastrugi (wind-induced feature) components. The proposed model adapts and extends the Freeman and Durden decomposition for an ice volume scenario in which the volume is a dielectric medium. Forms of the model for both random and oriented volumes are considered, and a new oriented sastrugi component is introduced which is able to explain backscatter behavior between different winter scenes. Validation is performed with airborne PolSAR data at $L$ - and P-band collected using the E-SAR system of the German Aerospace Center over the Austfonna ice cap in Svalbard, Norway, as part of the ICESAR campaign.
\end{abstract}

Index Terms-Land ice, polarimetric synthetic aperture radar (PolSAR), scattering mechanism, target decomposition.

\section{INTRODUCTION}

$\mathbf{S}$ YNTHETIC aperture radar (SAR) is a powerful remote sensing tool with which to monitor glaciers and ice sheets due to its high spatial resolution and wide coverage and its ability to penetrate beneath the ice surface to observe subsurface structure. However, owing to the medium's complexity, SAR

Manuscript received December 11, 2009; revised March 26, 2010 and May 12, 2010; accepted June 6, 2010. Date of publication August 26, 2010; date of current version December 27, 2010. This work was supported in part by the Microwaves and Radar Systems Institute of the German Aerospace Center (DLR), by the German Academic Exchange Service (DAAD), and by the European Space Agency under Contract 20655/07/NL/CB.

J. J. Sharma and A. Moreira are with the Microwaves and Radar Institute, German Aerospace Agency (DLR), 82230 Wessling, Germany, and also with the University of Karlsruhe, 76128, Karlsruhe, Germany (e-mail: jayanti.sharma@dlr.de; alberto.moreira@dlr.de).

I. Hajnsek is with the Microwaves and Radar Institute, German Aerospace Agency (DLR), 82230 Wessling, Germany, and also with the Institute of Environmental Engineering, ETH Zürich, 8093 Zurich, Switzerland (e-mail: irena.hajnsek@dlr.de).

K. P. Papathanassiou is with the Microwaves and Radar Institute, German Aerospace Agency (DLR), 82230 Wessling, Germany (e-mail: kostas.papathanassiou@dlr.de).

Color versions of one or more of the figures in this paper are available online at http://ieeexplore.ieee.org.

Digital Object Identifier 10.1109/TGRS.2010.2056692 backscattering from ice remains poorly understood, including the relative importance of scattering from surface and volume layers and also dependences on frequency and glacier zone (facie).

The radar signal is a function of the material and structural properties of the illuminated scene, including volume and surface backscatter at various roughness scales. Due to extreme and variable weather conditions, surface properties of glacier ice such as surface roughness and wind-induced features can change quickly over a period of days or even hours [1]. In addition, features correlating with wind direction can display considerable orientation [2]. As polarimetry is sensitive to the shape and orientation of the scatterer, these effects can have a significant influence upon observed polarimetric SAR (PolSAR) signatures. To infer geophysical properties of the underlying ice volume such as extinction and density, separation of the ground and volume contributions becomes necessary.

The objective of this paper is to investigate how well the volume response can be isolated using decomposition techniques in order to separate the radar return into several scattering components. Cloude and Pottier [3] identified three main types of target decomposition theorems: those employing a coherent decomposition of the scattering matrix, those using an eigenvector analysis of the covariance or coherency matrix, and those modeling the covariance or coherency matrix. Decompositions of the scattering matrix are well suited for high-resolution lowentropy scattering problems [3], in which scattering is due to a few dominant scattering centers, but are generally inappropriate for modeling distributed targets such as ice volumes. Similarly, eigendecomposition provides an interpretation of the coherency matrix in terms of orthogonal rank-one elementary scattering processes, which are ill suited for describing ice scattering at long wavelengths. For these reasons, a model-based approach is used in this paper.

For natural terrain, Freeman and Durden proposed a threecomponent scattering model, decomposing the measured covariance matrix into surface, dihedral, and volume scattering contributions [4]. Extensions of this decomposition have been published for urban [5], wetland [6], and agricultural applications [7]. However, no polarimetric decompositions of the covariance matrix have been published for glacier ice, primarily because of a lack of experimental data.

PolSAR results in the ice literature are limited to those using airborne sensors over Greenland [8], [9] and also results using SIR-C and AIRSAR data in the Alps, Tibet, and the Andes [10]-[13]. The focus to date has been on descriptions of polarimetric properties [8], [12], [13] and discrimination between snow and bare ice areas [10], [11] rather than modeling. Rignot 
[9] developed a backscatter model for the percolation zone in Greenland, where ice inclusions were modeled as dielectric cylinders embedded in a transparent snow medium and surface scattering was not included in the model. The agreement between modeled and measured backscatter was fairly good at C-band; however, comparisons at L- and P-band were poorer.

Interferometric SAR (InSAR) provides additional information on the vertical distribution of scatterers in the ice. C-band InSAR observables have been used to parameterize the relation between interferometric coherence and penetration depth [14], and snow accumulation [15]. A common problem is the separation of ground (surface) and volume contributions that requires the use of a scattering model. Furthermore, long satellite revisit times induce temporal decorrelation and limit the use of InSAR to periods without intervening precipitation, wind, or melt events. Polarimetric interferometry (Pol-InSAR) using airborne sensors has been used to investigate glaciers and ice sheets at L- and P-band [16], [17], although again, a model is required to separate the individual scattering contributions and to interpret results. The deep penetration of the signals at these longer wavelengths complicates both modeling and interpretation.

As a first step and to limit the complexity of the problem, a polarimetric model of glacier ice backscatter is considered in this work. A new polarimetric decomposition is proposed, modeling PolSAR observables as a combination of contributions from a slightly rough surface (at the snow-ice interface), a volume response from a potentially oriented particle cloud, and an oriented wind-induced surface sastrugi field. Since ice volumes may often be expected to have a preferential orientation of scatterers, particularly horizontal stratification and layering, such an extension is important for quantitative remote sensing inversion. A unique fully polarized airborne SAR data set is available for validation acquired at L- and P-band and with a one-month temporal baseline to investigate the temporal evolution of polarimetric properties.

Section II presents scattering models for surface, volume, and sastrugi components. The combined model and decomposition approach, as well as simulations demonstrating its sensitivity, is outlined in Section III. Experimental data collected at L- and P-band using the E-SAR system of the German Aerospace Center (DLR) and their polarimetric signatures are described in Section IV and are used in Section V to perform polarimetric decomposition. A discussion of the results is provided in Section VI, and conclusions and recommendations are given in Section VII.

\section{Scattering Contributions Over Glacier ICE}

This section reviews several elementary scattering mechanisms which may be of importance in polarimetric modeling of land ice. Electromagnetic models are developed for long-wavelength microwaves, e.g., L- and P-band, with center frequencies of 1.3 and $0.35 \mathrm{GHz}$, respectively. At these wavelengths, surface as well as volume contributions can be expected. Winter and early spring conditions without melt are assumed.

Natural scenes contain many spatially distributed deterministic scattering centers, each of which may be represented by an individual scattering matrix in the HV basis as [18]-[20]

$$
\boldsymbol{S}:=\left[\begin{array}{ll}
S_{\mathrm{HH}} & S_{\mathrm{HV}} \\
S_{\mathrm{VH}} & S_{\mathrm{VV}}
\end{array}\right] .
$$

For the monostatic case, with backscattering from reciprocal media such that $S_{\mathrm{HV}}=S_{\mathrm{VH}}$, the response from a single deterministic particle is completely described by a three-element lexicographic scattering vector $\vec{k}_{L}=\left[S_{\mathrm{HH}}, \sqrt{2} S_{\mathrm{HV}}, S_{\mathrm{VV}}\right]^{\mathrm{T}}$, where superscript $\mathrm{T}$ represents the matrix transpose.

For distributed targets, it is necessary to evaluate the secondorder statistics of the scattering matrix, and thus, the polarimetric covariance matrix $C^{H V}$ is introduced. It is formed from the outer product of $\vec{k}_{L}$ with its conjugate transpose [20]-[22]

$$
\begin{aligned}
\boldsymbol{C}^{\boldsymbol{H} \boldsymbol{V}} & :=\left\langle\vec{k}_{L} \vec{k}_{L}^{\dagger}\right\rangle \\
& =\left[\begin{array}{ccc}
\left\langle\left|S_{\mathrm{HH}}\right|^{2}\right\rangle & \sqrt{2}\left\langle S_{\mathrm{HH}} S_{\mathrm{HV}}^{*}\right\rangle & \left\langle S_{\mathrm{HH}} S_{\mathrm{VV}}^{*}\right\rangle \\
\sqrt{2}\left\langle S_{\mathrm{HV}} S_{\mathrm{HH}}^{*}\right\rangle & 2\left\langle\left|S_{\mathrm{HV}}\right|^{2}\right\rangle & \sqrt{2}\left\langle S_{\mathrm{HV}} S_{\mathrm{VV}}^{*}\right\rangle \\
\left\langle S_{\mathrm{VV}} S_{\mathrm{HH}}^{*}\right\rangle & \sqrt{2}\left\langle S_{\mathrm{VV}} S_{\mathrm{HV}}^{*}\right\rangle & \left\langle\left|S_{\mathrm{VV}}\right|^{2}\right\rangle
\end{array}\right]
\end{aligned}
$$

where $\nmid$ denotes the conjugate transpose, $*$ denotes the complex conjugate, and $\langle$.$\rangle denotes the ensemble average.$

\section{A. Surface Scattering}

The well-documented decrease in backscatter $\left(\sigma^{0}\right)$ with increasing incidence angle over land ice at microwave frequencies ranging from $\mathrm{X}$ - to L-bands [10], [13] suggests the presence of a surface scattering component. Surface scatter at L- and P-band wavelengths is postulated to originate from the snow-ice interface and to conform to the first-order small perturbation method (SPM) for a slightly rough interface.

Although, in general, surface roughness is depolarizing, the surface or ground covariance matrix $\boldsymbol{C}_{\boldsymbol{g}}^{\boldsymbol{H} \boldsymbol{V}}$ is modeled as a rank-one reflection symmetric matrix with zero crosspolar response through the use of the SPM [1]

$$
\boldsymbol{C}_{\boldsymbol{g}}^{\boldsymbol{H} \boldsymbol{V}}=f_{g}\left[\begin{array}{ccc}
|\beta|^{2} & 0 & \beta \\
0 & 0 & 0 \\
\beta^{*} & 0 & 1
\end{array}\right]
$$

where

$$
\beta=\frac{R_{h}}{R_{v}} e^{j \phi_{\beta}} .
$$

In (3) and (4), $f_{g}$ is the ground power coefficient, $R_{h}$ and $R_{v}$ are the Bragg coefficients for horizontally and vertically polarized waves, $j$ is the imaginary unit, and $\phi_{\beta}$ is a copolar phase component. $R_{h}$ and $R_{v}$ are given in [23], where, in this paper, the relative permittivity $\varepsilon_{r}$ is replaced by $\varepsilon_{2 r} / \varepsilon_{1 r}$, representing the ratio of the relative permittivities in the second medium (ice) and the first medium (snow), and the incidence angle is replaced by the angle in the snow after refraction according to Snell's law.

As opposed to [4], $|\beta|$ is fixed to conform to the SPM using knowledge of the approximate snow and ice dielectric constants, although the unknown phase component $\phi_{\beta}$ is retained. 


\section{B. Ice Volume Scattering}

Modeling the ice volume component is challenging due to the complex electromagnetic interactions involved, the lack of in situ measurements, and test-site-dependent variations in ice structure, such that the dominant scatterers can be ice crystals [24], ice inclusions (ice pipes or lenses) [9], buried sastrugi, or layers [15]. Assuming that one of the first three aforementioned components dominates the volume return, the volume is modeled as a homogeneous cloud of identically shaped and sized scatterers [4], [25]. Scattering is principally controlled by three factors: the density and extinction properties of the volume (affecting the scattered power), the particle shape, and the orientation distribution of the particles. Up to an amplitude factor, the scattering matrix of a single spheroid in a local coordinate system aligned parallel to the axes of symmetry of the particle can be given by [21]

$$
\boldsymbol{S}_{\text {spheroid }}=\left[\begin{array}{cc}
1 & 0 \\
0 & A_{p}
\end{array}\right]
$$

where $A_{p}$ is the ratio of the principal polarizabilities. For simplicity and to reduce the number of unknowns, the scatterers are assumed to be thin dipoles as in [1] for which $A_{p}=0$. The influence of scatterer orientation - of significant interest in this paper-is maximized with a dipole particle shape. A more generalized treatment of the scattering matrix for the spheroid case can be found in [25] and [26].

Particle orientations can be simulated as being completely random [1], in which case the volume is azimuthally symmetric, or as being oriented [5], [25], where orientation distributions are typically referenced to the plane perpendicular to the line of sight (LOS). To avoid the implications of a variation in LOS induced by the inherent variation of incidence angle in airborne SAR systems, an Earth-based coordinate system is chosen as a reference in this work.

1) Oriented Volume: A polarimetric decomposition is proposed in which the volume of scatterers is permitted to have an orientation. It is assumed that topographic variations are negligible, which is reasonable for ice caps and sheets which are relatively flat. To begin, we assume the simplest possible case where the incident field intensity remains the same everywhere in the volume and only single scattering is considered (Born approximation).

We define a coordinate system with axes $(x, y, z)$, where the $z$-axis is normal to the Earth's surface, $x$ is along the line of flight (i.e., azimuth), and $y$ is in the ground-range direction (perpendicular in a right-handed system). Let us further write $(x, y, z)$ in a spherical coordinate system defined by angles $(\nu, \psi)$ and radius $r$ such that

$$
\left[\begin{array}{c}
x \\
y \\
z
\end{array}\right]=r\left[\begin{array}{c}
\cos \nu \cos \psi \\
\sin \nu \cos \psi \\
\sin \psi
\end{array}\right]
$$

where $-\pi / 2 \leq \psi \leq \pi / 2$ and $0 \leq \nu \leq 2 \pi$, as shown in Fig. 1 . The radar geometry is defined by a new coordinate system $\left(x^{\prime}, y^{\prime}, z^{\prime}\right)$ created by a rotation about the $x$-axis by $-\delta$, where $\delta$ is the complement of the incidence angle $\left(\delta=\pi / 2-\theta_{\text {inc }}\right)$. Note that, for glacier applications, the incidence angle $\theta_{\text {inc }}$

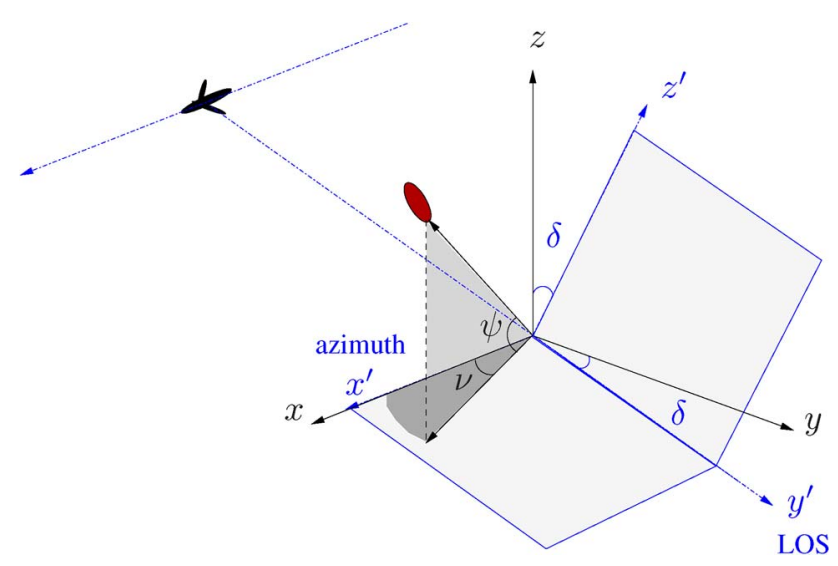

Fig. 1. Relation between Earth-based $(x, y, z)$ and radar geometry $\left(x^{\prime}, y^{\prime}, z^{\prime}\right)$ coordinate systems. Orientation of a single particle is shown, described by orientation angles $(\nu, \psi) . \delta\left(=\pi / 2-\theta_{\text {inc }}\right)$ is a rotation about the $x$-axis.

must be replaced by the refracted angle in the medium $\theta_{r}$ as dictated by Snell's law. LOS is along the $y^{\prime}$-axis, and the plane perpendicular to the LOS is defined by the $x^{\prime} z^{\prime}$-plane. The horizontal polarization $h$-axis is along the line of flight, and the vertical polarization $v$-axis is parallel to $z^{\prime}$. The relation between $(x, y, z)$ and $\left(x^{\prime}, y^{\prime}, z^{\prime}\right)$ is then

$$
\begin{aligned}
{\left[\begin{array}{l}
x^{\prime} \\
y^{\prime} \\
z^{\prime}
\end{array}\right] } & =\left[\begin{array}{ccc}
1 & 0 & 0 \\
0 & \cos (-\delta) & \sin (-\delta) \\
0 & -\sin (-\delta) & \cos (-\delta)
\end{array}\right]\left[\begin{array}{l}
x \\
y \\
z
\end{array}\right] \\
& =r\left[\begin{array}{c}
\cos \nu \cos \psi \\
\sin \nu \cos \psi \cos \delta-\sin \psi \sin \delta \\
\sin \nu \cos \psi \sin \delta+\sin \psi \cos \delta
\end{array}\right] .
\end{aligned}
$$

Considering a single dipole, the equivalent scattering matrix observed by a monostatic radar for this particle can be expressed as a function of $\nu, \psi$, and $\delta$ given its projection onto the $h$ - and $v$-axes from (7). It is assumed that the distance between the sensor and the volume of particles is very large such that, $r$ [see (7)] can be considered constant for all particles within the resolution cell. The scattering matrix is then

$$
\boldsymbol{S}_{\text {dipole }}(\nu, \psi, \delta)=\left[\begin{array}{ll}
S_{\mathrm{HH}} & S_{\mathrm{HV}} \\
S_{\mathrm{VH}} & S_{\mathrm{VV}}
\end{array}\right]
$$

where

$$
\begin{aligned}
S_{\mathrm{HH}} & =\cos ^{2}(\nu) \cos ^{2}(\psi) \\
S_{\mathrm{VV}} & =(\sin \nu \cos \psi \sin \delta+\sin \psi \cos \delta)^{2} \\
S_{\mathrm{HV}} & =S_{\mathrm{VH}} \\
& =\cos \nu \cos \psi(\sin \nu \cos \psi \sin \delta+\sin \psi \cos \delta) .
\end{aligned}
$$

Except for a scaling factor, the backscatter response from a single deterministic particle is completely described by $S$ in (8). To determine the superposition of responses from a distribution of particles within the volume, the covariance matrix is computed.

Assuming $\nu$ and $\psi$ to be independent variables with probability density functions (pdfs) $p(\nu)$ and $p(\psi)$, respectively, the expected value for each element $m n$ of the volume covariance matrix can be determined through integration of the pdfs over 


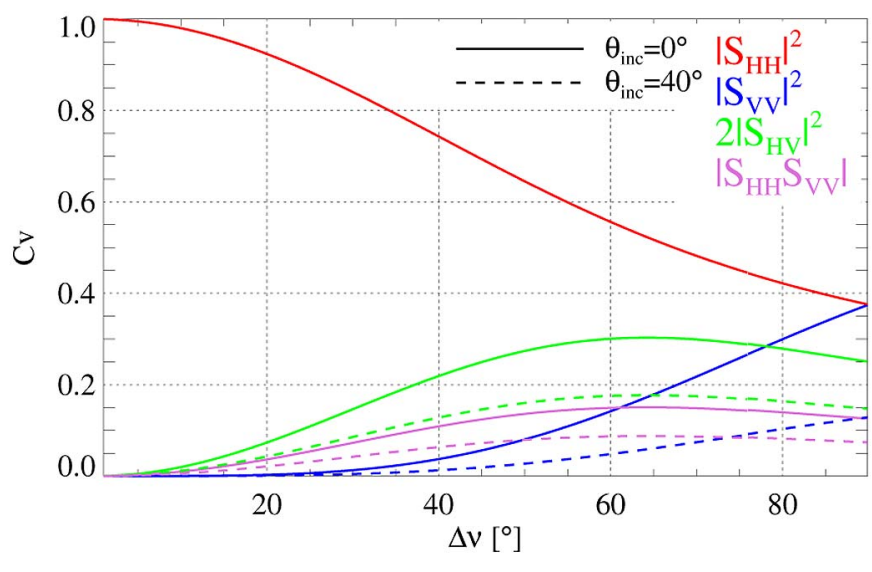

Fig. 2. Normalized covariance matrix elements for simulations of an oriented volume versus distribution width for $\nu_{0}=0^{\circ}$ and fixed $\psi=0^{\circ}$. Results at both nadir (solid) and $\theta_{\mathrm{inc}}=40^{\circ}$ (dashed) are given. The $\left|S_{\mathrm{HH}}\right|^{2}$ response at both incidence angles coincides.

all possible orientation angles and for each linear polarization combination $i j$

$$
\left\langle C_{v m n}\right\rangle=F_{m n} \int_{0}^{2 \pi} \int_{-\pi / 2}^{\pi / 2} S_{i} S_{j}^{*} \cos \psi p(\psi) p(\nu) d \psi d \nu
$$

where the $\cos \psi$ term is required for a uniform spatial distribution of scatterers and $F_{m n}$ is the factor of 1,2 or $\sqrt{2}$ for each element from (2) required to conserve total power.

Various distribution functions $p(\psi)$ and $p(\nu)$ are possible. Results from simulations in which $\psi$ is fixed to zero and $\nu$ is modeled as a uniform distribution centered at $\nu_{0}$ with width $2 \Delta \nu$, with $\Delta \nu$ varied from $0^{\circ}$ to $90^{\circ}$, are shown in Fig. 2. After [25], pdf $p(\nu)$ is

$$
\frac{1}{p(\nu)}= \begin{cases}\frac{1}{2 \Delta \nu}, & \text { for } \nu_{0}-\Delta \nu \leq \nu \leq \nu_{0}+\Delta \nu \\ 0, & \text { otherwise. }\end{cases}
$$

To obtain Fig. 2, the integrals from (9) were evaluated and $\boldsymbol{C}_{\boldsymbol{v}}$ components were determined as a function of $\Delta \nu$ for $\nu_{0}=0$. Results are plotted for both nadir $\left(\theta_{\text {inc }}=0^{\circ}\right)$ and an incidence angle of $40^{\circ}$. Results at $\theta_{\text {inc }}=0^{\circ}$ are identical to those using the model given in [25] for zero tilt, such that scatterers are assumed to be distributed in the plane perpendicular to the LOS. However, as incidence angle is increased, it is seen that backscatter at VV and $\mathrm{HV}$ differs significantly from that predicted using the conventional 2-D model. For this reason, a 3-D model is required for accurate modeling. As mentioned earlier, many possibilities exist in choosing $p(\psi)$ and $p(\nu)$. Other pdfs such as truncated Gaussian or circular normal distributions [26] are also possible and may provide a better match to the actual scattering physics, although they are more mathematically involved and display deviations of $\lesssim 20 \%$ from results assuming a truncated uniform distribution [27]. It is seen in Fig. 2 that the model predicts larger $\left|S_{\mathrm{HH}}\right|^{2}$ than $\left|S_{\mathrm{VV}}\right|^{2}$ except at $\Delta \nu=90^{\circ}$ which represents completely random scattering for a nadirlooking system. Variation in $\Delta \nu$ can be used to model a wide range of $\left|S_{\mathrm{HH}}\right|^{2} /\left|S_{\mathrm{VV}}\right|^{2}$ polarization ratios, which is necessary to reconstruct the polarimetric properties seen in experimental data.
In the simplifying case of a completely random volume such that $p(\nu)=1 /(2 \pi)$ and $p(\psi)=1 / \pi$ in (9), the volume covariance matrix reduces to the reflection symmetric form given in [4]

$$
\boldsymbol{C}_{v}^{\boldsymbol{H} \boldsymbol{V}}=f_{v}\left[\begin{array}{ccc}
1 & 0 & \frac{1}{3} \\
0 & \frac{2}{3} & 0 \\
\frac{1}{3} & 0 & 1
\end{array}\right]
$$

2) Oriented Volume With Propagation Effects: In order to model a more realistic scenario, extinction and refraction effects are introduced. As a wave propagates through the volume, it is attenuated due to absorption and scattering and refracted due to the dielectric constant. If attenuation and refraction are isotropic, i.e., polarization independent, their effects may be absorbed into the $f_{v}$ power coefficient. However, in the case of oriented volumes (due to, e.g., oriented ice inclusions or ice crystals oriented to conform to the prevailing stress or elongated in the direction of a thermal gradient [28]), differential extinction and refractivity must be considered. Extinction consists of both scattering and absorption contributions, and polarization dependences can be attributed to scattering from oriented features and/or to polarization-dependent absorption due to an orientation dependence of the imaginary part of the ice dielectric constant [29]. Differential refractivity (i.e., directional dependence of the real part of the dielectric constant) can arise from anisotropy in the dielectric constant of oriented ice crystals [30], [31] or from density variations at shallow depths if oriented scatterers such as sastrugi and dunes are preserved within the ice [32].

A wave incident at an arbitrary polarization will change its polarization as it propagates through the volume except at the eigenpolarizations $a$ and $b$, and thus, computations involving orientation propagation effects will be carried out in this basis. The eigenpolarizations are given by the eigenvectors of the average forward-scattering matrix which is assumed to be symmetric such that the eigenvectors are orthogonal [33].

We begin with a general scenario in which the $h$ and $v$ polarization axes (measured by the radar) do not necessarily correspond to the eigenpolarizations. Let us define $\zeta$ as the angle between the $h$-axis in the radar geometry and the mean orientation unit vector of the volume ((6) divided by its norm) projected to the plane perpendicular to the LOS. From (7)

$$
\zeta=\tan ^{-1}\left(\frac{\sin \nu_{0} \cos \psi_{0} \sin \delta+\sin \psi_{0} \cos \delta}{\cos \nu_{0} \cos \psi_{0}}\right) .
$$

To transform between the HV polarization basis and the eigenpolarization AB basis, unitary rotation matrix $R$ is defined [34]

$$
\boldsymbol{R}(2 \zeta)=\frac{1}{2}\left[\begin{array}{ccc}
1+\cos (2 \zeta) & \sqrt{2} \sin (2 \zeta) & 1-\cos (2 \zeta) \\
-\sqrt{2} \sin (2 \zeta) & 2 \cos (2 \zeta) & \sqrt{2} \sin (2 \zeta) \\
1-\cos (2 \zeta) & -\sqrt{2} \sin (2 \zeta) & 1+\cos (2 \zeta)
\end{array}\right] .
$$

The total volume covariance matrix in the $\mathrm{AB}$ basis is then

$$
\boldsymbol{C}_{\boldsymbol{v}}^{\boldsymbol{A B}}=\boldsymbol{R}(2 \zeta) \boldsymbol{C}_{\boldsymbol{v}}^{\boldsymbol{H} \boldsymbol{V}} \boldsymbol{R}(2 \zeta)^{\mathrm{T}}
$$

where the polarization basis is given by the superscript. 
Assuming a homogeneous exponentially lossy and infinite volume (appropriate for microwave frequencies over glacier ice), the covariance matrix in the $\mathrm{AB}$ basis can be described by

$$
\boldsymbol{C}_{\boldsymbol{v}}^{\boldsymbol{A B}}=\int_{-\infty}^{0} e^{\frac{\left(\kappa_{a}+\kappa_{b}\right) z}{\cos \theta_{r}}} \boldsymbol{P}^{\boldsymbol{A B}}(z) \tilde{\boldsymbol{C}}_{\boldsymbol{v}}^{\boldsymbol{A B}} \boldsymbol{P}^{\boldsymbol{A} \boldsymbol{B}^{\dagger}}(z) d z .
$$

In the aforementioned equation, $P^{A B}(z)$ is the propagation matrix in the eigenbasis $\mathrm{AB}$

$$
\boldsymbol{P}^{A B}(z)=\left[\begin{array}{ccc}
e^{\tau} & 0 & 0 \\
0 & 1 & 0 \\
0 & 0 & e^{-\tau}
\end{array}\right]
$$

where $\boldsymbol{P}^{A B}(z)$ has been transformed from [33] into its covariance matrix form. In (16), $\tau$ is the complex differential propagation constant given by [33] which has been modified here for a volume under ground $(z \leq 0)$ to be

$$
\tau=\left(-\left(\frac{\kappa_{a}-\kappa_{b}}{2}\right)+j k\left(\chi_{a}-\chi_{b}\right)\right) \frac{-z}{\cos \theta_{r}}
$$

where $\kappa_{a}$ and $\kappa_{b}$ are the extinction coefficients, $k=2 \pi / \lambda$ is the wavenumber, and $\chi_{a}$ and $\chi_{b}$ are the refractivities of the eigenpolarizations. Let $\Delta \chi=\left(\chi_{a}-\chi_{b}\right)$ represent the difference in refractivity between the eigenpolarizations.

Matrix $\tilde{C}_{v}^{A B}$ in (15) accounts for the geometric projection of the distribution of scatterer orientations onto the $a$ - and $b$ axes. This matrix is determined by evaluating elements of the volume covariance matrix from (9) and projecting them into the $\mathrm{AB}$ basis using (14). Evaluating the integral in (15), one can determine $C_{v}^{A B}$ as a function of $\tilde{C}_{v}^{A B}$

$$
\boldsymbol{C}_{\boldsymbol{v}}^{\boldsymbol{A B}}=f_{v}\left[\begin{array}{ccc}
C_{v 11}^{\mathrm{AB}} & C_{v 12}^{\mathrm{AB}} & C_{v 13}^{\mathrm{AB}} \\
C_{v 12}^{\mathrm{AB} *} & C_{v 22}^{\mathrm{AB}} & C_{v 23}^{\mathrm{AB}} \\
C_{v 13}^{\mathrm{AB} *} & C_{v 23}^{\mathrm{AB} *} & C_{v 33}^{\mathrm{AB}}
\end{array}\right]
$$

where

$$
\begin{aligned}
C_{v 11}^{\mathrm{AB}} & =\tilde{C}_{v 11}^{\mathrm{AB}} \frac{\cos \theta_{r}}{2 \kappa_{a}} \\
C_{v 22}^{\mathrm{AB}} & =\tilde{C}_{v 22}^{\mathrm{AB}} \frac{\cos \theta_{r}}{\kappa_{a}+\kappa_{b}} \\
C_{v 33}^{\mathrm{AB}} & =\tilde{C}_{v 33}^{\mathrm{AB} \frac{\cos \theta_{r}}{2 \kappa_{b}}} \\
C_{v 13}^{\mathrm{AB}} & =\tilde{C}_{v 13}^{\mathrm{AB}} \frac{\cos \theta_{r}}{\left(\left(\kappa_{a}+\kappa_{b}\right)-j 2 k \Delta \chi\right)} \\
C_{v 12}^{\mathrm{AB}} & =\tilde{C}_{v 12}^{\mathrm{AB}} \frac{2 \cos \theta_{r}}{\left(\left(3 \kappa_{a}+\kappa_{b}\right)-j 2 k \Delta \chi\right)} \\
C_{v 23}^{\mathrm{AB}} & =\tilde{C}_{v 23}^{\mathrm{AB}} \frac{2 \cos \theta_{r}}{\left(\left(\kappa_{a}+3 \kappa_{b}\right)-j 2 k \Delta \chi\right)} .
\end{aligned}
$$

In the instance of an infinite volume, the copolar phase difference in the $\mathrm{AB}$ basis (phase of element $C_{v 13}^{\mathrm{AB}}$ ) reduces to

$$
\phi_{\mathrm{AA}-\mathrm{BB}}=\tan ^{-1}\left(\frac{2 k \Delta \chi}{\kappa_{a}+\kappa_{b}}\right)
$$

where $-90^{\circ} \leq \phi_{\mathrm{AA}-\mathrm{BB}} \leq 90^{\circ}$.

Assuming a uniform angular distribution of equally sized scatterers in the Earth $x y$-plane (Fig. 1) results (in general) in a nonuniform scatterer distribution when projected to the plane perpendicular to the LOS, violating reflection symmetry. However, $C_{v}^{A B}$ becomes reflection symmetric for certain combinations of $\nu, \psi$, and $\delta$, including as follows: a nadir incidence angle $\left(\delta=90^{\circ}\right)$, for $\nu_{0}=90^{\circ}$, for $\nu_{0}=0^{\circ}$ and $\psi_{0}=0^{\circ}$, for fixed $\psi$ and $\nu$ (i.e., $\Delta \nu=0$ and $\Delta \psi=0$ ), or for a completely random particle distribution (i.e., $\Delta \nu=\Delta \psi=90^{\circ}$ ) as in (11).

Lastly, the volume covariance matrix is transformed back into the HV basis through inversion of (14) to yield

$$
\boldsymbol{C}_{\boldsymbol{v}}^{\boldsymbol{H} \boldsymbol{V}}=\boldsymbol{R}(2 \zeta)^{\mathrm{T}} \boldsymbol{C}_{\boldsymbol{v}}^{\boldsymbol{A B}} \boldsymbol{R}(2 \zeta) .
$$

3) Transmissivities: Transmissivities are integrated into the aforementioned model, as is required for dielectric media such as ice and snow. In this paper, the relative dielectric constant $\varepsilon_{r}$ was computed from an empirical relation to firn density [35], where firn is defined as dense snow which has survived at least one summer. Approximate firn and snow densities obtained from in situ data of 0.8 and $0.4 \mathrm{~g} / \mathrm{cm}^{3}$, respectively, yield relative permittivities $\varepsilon_{r \text { firn }}=2.8$ and $\varepsilon_{r \text { snow }}=1.7$. For simplicity and due to a lack of depth-density profiling, homogeneous snow and firn media are assumed.

Negligible reflection at the air-snow boundary [23] and no scattering within the snowpack are presumed, which are reasonable for dry snow at L- and P-band. At the snow-firn interface, it is assumed that the surface is relatively smooth with respect to the wavelength and transmissivities are approximated using the Fresnel equations, where transmission coefficients $T_{h}$ (for horizontal polarization) and $T_{v}$ (for vertical polarization) are given in [36]. Accounting for transmission yields a slightly modified version of the volume covariance matrix

$\boldsymbol{C}_{\boldsymbol{v} \text { trans }}^{\boldsymbol{H} \boldsymbol{V}}=\boldsymbol{C}_{\boldsymbol{v}}^{\boldsymbol{H} \boldsymbol{V}} \circ\left[\begin{array}{ccc}\Upsilon_{h}^{2} & \Upsilon_{h} T_{h 21} T_{v 21} & \Upsilon_{h} \Upsilon_{\boldsymbol{v}} \\ \Upsilon_{h} T_{h 21} T_{v 21} & \Upsilon_{h} \Upsilon_{\boldsymbol{v}} & T_{h 21} T_{v 12} \Upsilon_{\boldsymbol{v}} \\ \Upsilon_{h} \Upsilon_{\boldsymbol{v}} & T_{h 21} T_{v 12} \Upsilon_{\boldsymbol{v}} & \Upsilon_{\boldsymbol{v}}^{2}\end{array}\right]$

where

$$
\Upsilon_{h}=T_{h 12} T_{h 21} \quad \Upsilon_{v}=T_{v 12} T_{v 21}
$$

and where $\circ$ represents element-wise multiplication (Hadamard product), $C_{v}^{H V}$ represents the volume covariance matrix from (20), and subscripts 1 and 2 represent the snow and firn media, respectively.

\section{Oriented Sastrugi Field}

In addition to surface and volume scattering, a third relevant scattering mechanism is expected which arises from an oriented sastrugi field. As will be shown for the experimental data in Section IV-B, surface and volume scattering alone are insufficient to explain temporal variations in the copolar ratio in the absence of melt, necessitating a third component. Sastrugi are snow dunes formed by wind deposition and erosion of the snow surface [37] and are widespread across ice sheets with high winter accumulation rates and strong winds. These streamlined ridges of densely packed snow are generally oriented parallel to the prevailing wind direction [1], although the exact position and shape of single sastrugi are random. The dimensions of single sastrugi are typically a meter to a few meters in the horizontal direction and a few tens of centimeters in the vertical, 


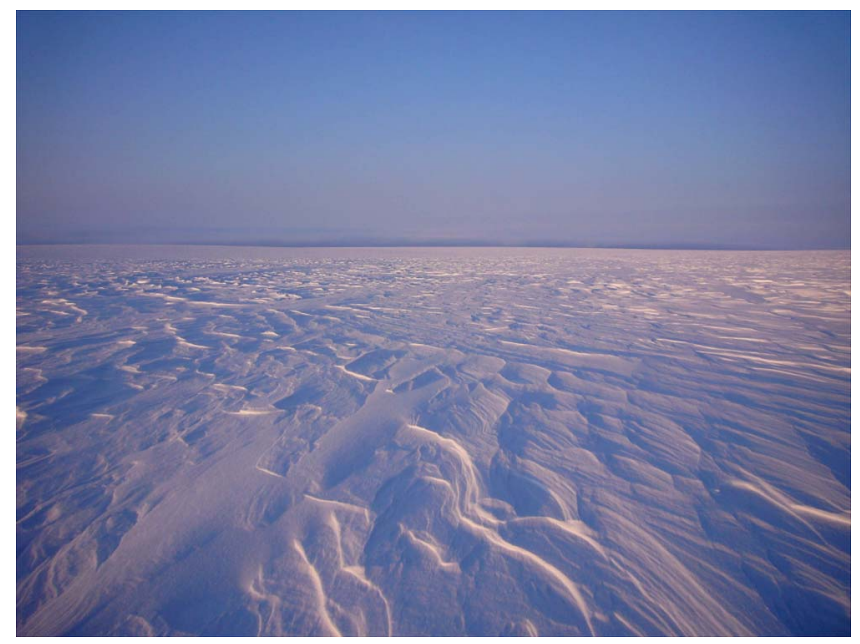

Fig. 3. Sastrugi on the Austfonna ice cap from the spring 2007 field season (photograph courtesy of Thorben Dunse, University of Oslo).

depending on wind strength, temperature, and snow hardness [37]. Sastrugi form rapidly in hours to days and, once hardened, can persist for months [2]. An example of sastrugi for the test site of interest (see Section IV-A) is given in Fig. 3.

Because of their orientation, sastrugi can have a strong impact on radar backscatter. Over Antarctica and Greenland, azimuth modulations of up to $\pm 5 \mathrm{~dB}$ in scatterometer data due to snow surface structure have been reported [2]. It is thus important to consider these features in modeling the return from glacier ice, particularly since sastrugi are of the same order as the L- and P-band wavelengths under consideration.

An oriented sastrugi field can be seen as a simplified form of the volume modeled in Section II-B1, where all scatterers are contained within a plane on the air-snow surface, and thus, there are no propagation effects. Assuming a truncated uniform distribution in $\nu$ and integrating (9) for fixed $\psi=0$ yield sastrugi covariance matrix

$$
\boldsymbol{C}_{\boldsymbol{s}}^{\boldsymbol{H} \boldsymbol{V}}=\frac{f_{\boldsymbol{s}}}{\Delta \nu}\left[\begin{array}{ccc}
f_{11} & f_{12} & f_{13} \\
f_{12} & 2 f_{13} & f_{23} \\
f_{13} & f_{23} & f_{33}
\end{array}\right]
$$

where

$$
\begin{aligned}
f_{11}= & 12 \Delta \nu+8 \cos \left(2 \nu_{0}\right) \sin (2 \Delta \nu)+\cos \left(4 \nu_{0}\right) \sin (4 \Delta \nu) \\
f_{12}= & 4 \sqrt{2}\left(\cos ^{4}\left(\Delta \nu-\nu_{0}\right)-\cos ^{4}\left(\Delta \nu+\nu_{0}\right)\right) \sin (\delta) \\
f_{13}= & \left(4 \Delta \nu-\cos \left(4 \nu_{0}\right) \sin (4 \Delta \nu)\right) \sin ^{2}(\delta) \\
f_{23}= & 4 \sqrt{2}\left(-\sin ^{4}\left(\Delta \nu-\nu_{0}\right)+\sin ^{4}\left(\Delta \nu+\nu_{0}\right)\right) \sin ^{3}(\delta) \\
f_{33}= & \left(12 \Delta \nu-8 \cos \left(\nu_{0}\right) \sin (2 \Delta \nu)\right. \\
& \left.\quad+\cos \left(4 \nu_{0}\right) \sin (4 \Delta \nu)\right) \sin ^{4}(\delta)
\end{aligned}
$$

and where $f_{s}$ is a scaling factor. Reflection symmetry about the plane containing the mean sastrugi orientation $\nu_{0}$ and the surface normal vector is assumed, but not with respect to the plane perpendicular to the LOS, such that $C_{s}^{H V}$ is not reflection symmetric. Accordingly, for the general case, the $C_{s}^{H V}$ matrix is fully populated, although for $\nu_{0}=0^{\circ}$ or $\nu_{0}=90^{\circ}$, or random $\nu$ (i.e., $\Delta \nu=90^{\circ}$ ), the terms representing cocross correlations [e.g., $\left\langle S_{\mathrm{HH}} S_{\mathrm{HV}}^{*}\right\rangle$ and $\left\langle S_{\mathrm{HV}} S_{\mathrm{VV}}^{*}\right\rangle$ from (2)] vanish, yielding a reflection symmetric sastrugi matrix of

$$
\boldsymbol{C}_{\boldsymbol{s}}^{\boldsymbol{H} \boldsymbol{V}_{\text {refl sym }}}=\frac{f_{s}}{\Delta \nu}\left[\begin{array}{ccc}
f_{11} & 0 & f_{13} \\
0 & 2 f_{13} & 0 \\
f_{13} & 0 & f_{33}
\end{array}\right] .
$$

\section{DECOMPOSITION APPROACH}

\section{A. Three-Component Decomposition for Glacier Ice}

Applying the results from the previous section, a polarimetric decomposition consisting of a surface (described by the SPM), a (possibly) oriented volume, and an oriented sastrugi field is proposed. Assuming that surface, volume, and sastrugi components are uncorrelated, the combined covariance matrix, expressed in (24), is a sum of the matrices for the individual mechanisms plus a diagonal noise matrix $N$. This additive (e.g., thermal) noise is modeled as a zero-mean Gaussian white noise process with noise power $n$ which is independent of the polarization state. It can be estimated using the decorrelation of the crosspolar channels as described in [38], and it is not treated as an unknown in the following analysis. In (24), the superscript $c$ denotes complex variables, and $g$ is ground (i.e., surface), $v$ is volume, and $s$ is sastrugi whose representations were given in (3), (21), and (22), respectively. Scattering power components of the ground $P_{g}$, volume $P_{v}$, and sastrugi $P_{s}$ are given by the trace of the corresponding covariance matrices.

$$
\begin{aligned}
\boldsymbol{C}_{\text {total }}^{\boldsymbol{H} \boldsymbol{V}}= & \underbrace{\left[\begin{array}{ccc}
g_{11} & 0 & g_{13}^{c} \\
0 & 0 & 0 \\
g_{13}^{c *} & 0 & g_{33}
\end{array}\right]}_{\boldsymbol{C}_{\boldsymbol{g}}^{\boldsymbol{H}}}+\underbrace{\left[\begin{array}{lll}
v_{11} & v_{12}^{c} & v_{13}^{c} \\
v_{12}^{c *} & v_{22} & v_{23}^{c} \\
v_{13}^{c *} & v_{23}^{c *} & v_{33}
\end{array}\right]}_{\boldsymbol{C}_{\boldsymbol{v}}^{\boldsymbol{H}}} \\
+ & \underbrace{\left[\begin{array}{lll}
s_{11} & s_{12} & s_{13} \\
s_{12} & s_{22} & s_{23} \\
s_{13} & s_{23} & s_{33}
\end{array}\right]}_{\boldsymbol{C}_{\boldsymbol{s}}^{\boldsymbol{H}}}+\underbrace{\left[\begin{array}{ccc}
n & 0 & 0 \\
0 & n & 0 \\
0 & 0 & n
\end{array}\right]}_{\boldsymbol{N}} .
\end{aligned}
$$

Each scattering mechanism in (24) contributes to different elements of the combined covariance matrix. Surface scattering contributes to $\mathrm{HH}$ and VV powers and to the complex copolar correlation. An oriented volume contributes to all components (powers and complex correlations), although for a random volume, $\boldsymbol{C}_{\boldsymbol{v}}^{\boldsymbol{H} \boldsymbol{V}}$ assumes its simplified reflection symmetric form from (11) in which case cocross components vanish and all elements are real numbers. Sastrugi can account for an amplitude contribution to all elements although with no phases. The proposed model is able to interpret a nonreflection symmetric covariance matrix in the $\mathrm{HV}$ basis as the presence of either oriented volume and/or oriented sastrugi scattering.

Considering the most general situation in which all three scattering mechanisms are present, there are 13 unknowns, which are listed in Table II in the Appendix. To distinguish volume orientations from sastrugi orientations, the subscript $s$ has been used for sastrugi. It is assumed that pdfs $p(\nu), p(\psi)$, and $p\left(\nu_{s}\right)$ are described by truncated uniform distributions, although the same number of unknowns would result for a Gaussian distribution, for instance. Incidence angle $\theta_{\text {inc }},|\beta|$, and transmissivities are assumed to be known quantities. 
The covariance matrix from (2) contains nine independent parameters. However, many natural media display reflection symmetry [39] for which the cocross correlations vanish and the covariance matrix contains only five nonzero elements, further reducing the number of radar observables. With 13 unknowns, (24) thus leads to an underdetermined inversion problem, and further simplifications and approximations are required.

First, we consider a scenario in which the sastrugi component is assumed to be negligible, reducing the number of unknowns to ten. However, parameters $\nu_{0}$ and $\psi_{0}$ are not well separated, and various combinations lead to the same $\zeta$ in (12). To avoid this ambiguity and as glacier ice is dominated by a horizontal layering due to melt patterns and buried wind-induced structures, a fixed $\psi=0$ is assumed such that all scatterers lie within the planes parallel to the Earth's surface. Eliminating $\psi_{0}$ and $\Delta \psi$, only eight unknowns remain, and with a fully populated covariance matrix (nine independent observables), it is possible to solve the system of equations. However, in the case of reflection symmetry (five observables), the problem remains underdetermined.

A second scenario is considered in which the volume particles are sufficiently randomly distributed such that $C_{v}^{H V}$ can be approximated by (11) (although transmissivities should be accounted for according to (21)). In this case, the only unknown parameter describing the volume component is $f_{v}$, and six unknowns remain. Because there are no propagation effects, cocross phase elements should be zero, and the covariance matrix contains seven independent parameters. Thus, there are sufficient degrees of freedom to solve. Reflection symmetry will arise if $\nu_{0 s}=0^{\circ}$ or $\nu_{0} s=90^{\circ}$; using a priori knowledge or copolar ratios, one could fix $\nu_{0} s$ to one of these two values, and the problem is still invertible for the reflection symmetric case.

The system of equations in (24) is solved numerically, where the solutions are constrained to be physically plausible, requiring positive powers, extinctions, and distribution widths. The optimization (cost) function was chosen to be the $L_{2}$ norm (sum of square residuals) of the set of nonzero covariance matrix elements.

\section{B. Simulation Results}

Simulated data are used to evaluate and validate the presented models and parameter retrieval techniques using the Monte Carlo method; correlations between polarizations are taken into account (see procedure in [22]), and fully developed speckle is assumed. For each scenario, polarimetric decomposition was performed on $512 \times 512$ independent realizations, and covariance matrices with 100 independent looks were used, balancing the need for spatial resolution while ensuring sufficient samples for a robust estimate.

Realistic values of snow and firn dielectric constants and surface roughness parameters were used from the literature. Volume scattering power coefficients $f_{v}$ 's were determined using HV magnitudes from the experimental data (Section IV). For oriented volumes, extinctions were set to $\kappa_{a}=0.25 \mathrm{~dB} / \mathrm{m}$ and $\kappa_{b}=0.2 \mathrm{~dB} / \mathrm{m}$, and differential refractivity $\Delta \chi$ was set to $20 \%$ of its maximum possible value when all ice crystals are in perfect alignment. A maximum $\Delta \chi$ of 0.01 was derived from the dielectric anisotropy of a single ice crystal in [30].

As described in Section III-A, not all model configurations can be inverted from a single covariance matrix, but the two cases discussed in Section III-A are applied to several scenarios.

1) Simulation of an oriented volume under ground (no sastrugi).

2) Simulation of a random volume under ground with sastrugi.

3) Consideration of the inversion of a random volume under ground with sastrugi exhibiting near-reflection symmetry. Scenario $3 \mathrm{a}$ inverts for all parameters, and $3 \mathrm{~b}$ assumes fixed $\nu_{0} s=0$.

4) Simulation of a random volume under ground with sastrugi exhibiting perfect reflection symmetry (fix $\nu_{0}$ ).

5) Simulation of an oriented volume under ground with sastrugi. Examining the impact of assuming a random volume in the presence of an oriented one.

For a given observed covariance matrix, the inverted parameters will be critically dependent upon the choice of scattering mechanisms included in the decomposition model.

To assess the stability of the inversion, simulations using both a priori knowledge to initialize parameters to their true values and without a priori knowledge were carried out. Tables III and IV in the Appendix list the modeling parameters and parameter retrieval performance, respectively, for the various scenarios at L-band at an incidence angle of $40^{\circ}$. By convention, power extinction coefficients are expressed in decibels per meter which may be converted to Nepers per meter through division by $10 \log _{10} e$. Normalized powers are quoted where the sum of all contributions is unity $\left(P_{g}^{n}+P_{v}^{n}+P_{s}^{n}=1\right)$. The percentage of pixels not meeting convergence criteria for each scenario is given in Table $\mathrm{V}$, also in the Appendix. For simulations at P-band, the relative amount of surface scattering $P_{g}^{n}$ was higher, and the normalized volume scattering contribution $P_{v}^{n}$ was lower than at L-band due to low HV magnitudes; smaller extinctions were used in the simulations in accordance with scattering physics. However, parameter estimation accuracies were very similar to those at L-band, and thus, for conciseness, they are omitted here.

Scenario 1 (oriented volume under ground):

1) If a priori values of the unknowns are given as initial parameters for the optimization, powers and volume orientation properties could be reliably estimated. However, $\kappa_{a}, \kappa_{b}$, and $\Delta \chi$ estimates have increased variances, i.e., display high sensitivity to speckle, suggesting ill conditioning. In addition, $\Delta \chi$ estimates are strongly biased.

2) When no a priori knowledge is available, the percentage of pixels for which no convergence is achieved increases substantially (Table V), and the bias in nearly all estimated parameters is significantly larger, indicating instability of the optimization and its sensitivity to local minima.

Scenario 2: If a random volume under ground is considered in the presence of sastrugi, inversion results are fairly accurate, and there is negligible sensitivity to the initial parameters. 
Scenario 3 (for which $\nu_{0} s=5^{\circ}$ generates a nearly reflection symmetric covariance matrix):

1) The power coefficients and distribution width are well estimated for $3 \mathrm{a}$, although there is a large bias in estimated $\nu_{0}$ s, even with a priori information. A smaller true $\nu_{0} s$ value leads to smaller cocross covariance elements in (22) and, thus, to increased speckle noise power for these elements which is mistaken for a large nonzero mean sastrugi orientation.

2) The inversion from 3 a suggests that if cocross correlation elements have low magnitudes, it may be preferable to fix $\nu_{0} s$ as in $3 \mathrm{~b}$, with more favorable inversion results.

Scenario 4: This scenario reveals the robust performance of the inversion algorithm in decomposing a random volume under ground if reflection symmetric sastrugi may be assumed.

Scenario 5: Because a single covariance matrix does not allow separation of ground, oriented volume, and oriented sastrugi components simultaneously, it is of interest to determine the decomposition performance when a random volume is assumed despite the presence of a certain degree of orientation.

1) The volume is characterized by a random distribution of orientation angles in $\nu\left(\Delta \nu=90^{\circ}\right)$, although $\psi$ is fixed to zero.

2) In Table IV, scenario 5 shows reasonable accuracies in estimating the power coefficients, although the distribution width $\Delta \nu_{s}$ is severely biased toward lower (more oriented) values to compensate for the assumption of a random volume. Thus, if an oriented volume is suspected to exist and the three-component decomposition with a random volume is applied, caution should be used in interpretation of the sastrugi orientation parameters.

A visual representation of parameter estimation using the simulated data without $a$ priori information is shown in Fig. 4. It is seen that scenario 1 has high uncertainties in extinction and $\Delta \chi$ parameters and that scenario $3 \mathrm{a}$ has a strongly biased $\nu_{0} s$ estimate. Common to all scenarios are low biases in power estimates, although variances in volume and sastrugi powers and sastrugi orientation are somewhat large. The best accuracies are obtained assuming reflection symmetry in scenarios $3 \mathrm{~b}$ and 4 . As expected, for all scenarios, inversion rates are higher when initial values lie close to the true parameters (Table V), although in all cases other than scenario 1 , there is a high rate of inversion $(>95 \%)$ without requiring a priori information and comparable accuracies to the a priori case. In summary, results indicate that inversion of the full-oriented volume can be unstable with large uncertainties, but that modeled powers and orientation characteristics can be reliably retrieved from the data in some cases, particularly when reflection symmetry can be assumed. Further performance improvements could be achieved by increasing the number of looks used for covariance matrix estimation (in simulations, standard deviations were roughly halved using 500 compared to 100 looks); this is accompanied by a significant loss in spatial resolution, which may, however, be acceptable over homogeneous ice sheets.

\section{EXPERIMENTAL DATA}

\section{A. Test Sites}

The test sites are located on the Austfonna ice cap, situated on the island of Nordaustlandet in northeastern Svalbard, Norway $\left(79.7^{\circ} \mathrm{N}, 24.0^{\circ} \mathrm{E}\right)$. Two sites were overflown, one in the firn zone near the summit of the ice cap (referred to as "Summit") and one in the superimposed ice (SI) zone near the Etonbreen outlet glacier ("Eton").

The sites have differing near-surface structure due to variation in summer melt. In the firn zone, meltwater percolates downward before refreezing into horizontally distributed ice layers or ice lenses and vertically distributed ice pipes, which can appear extremely bright in SAR imagery [8]. In the SI zone, meltwater freezes onto the cold glacier ice at the base of the snowpack, forming more homogeneous SI. Topography is very gentle with surface slopes of less than a few degrees at both sites.

\section{B. SAR Data and Ground Measurements}

The airborne SAR data were acquired as part of the ICESAR campaign in spring 2007, which was a joint project between the Microwaves and Radar Systems Institute of DLR and the Alfred-Wegener Institute, and supported by the European Space Agency. Fully polarized L-band $(1.3 \mathrm{GHz})$ and P-band $(350 \mathrm{MHz})$ data were collected using DLR's E-SAR system. Incidence angles varied from $25^{\circ}$ to $50^{\circ}$, and the data have a resolution of $2.1 \mathrm{~m} \times 1.0 \mathrm{~m}$ in slant range and azimuth, respectively. Data were acquired in March and April to investigate the temporal evolution of the polarimetric signatures.

Ground measurements were collected by a team from the University of Oslo and the Norwegian Polar Institute as part of CryoSat validation activities. Meteorological, snow stratigraphy, ground penetrating radar, and neutron probe data (from which density estimates were derived for Section II-B-3) were acquired during a two-week period in 2007, spanning the end of April and beginning of May prior to spring melt.

1) Polarimetric Signatures: In the following, a brief review of the polarimetric properties at the Summit and Etonbreen sites at both L- and P-band frequencies is given. A spatial averaging window of 100 effective looks was used to generate covariance matrices, corresponding to an approximately square window of $20 \mathrm{~m} \times 20 \mathrm{~m}$ in ground range. Calibration accuracies are estimated to be $\pm 1 \mathrm{~dB}$ for backscattering coefficients and $\pm 10^{\circ}$ for copolar phase imbalances.

Interpretation of SAR data over ice can be challenging as backscatter sources from the surface to the depth of penetration are integrated within one pixel. Fig. 5 shows composite Pauli RGB images which roughly indicate the presence of various scattering mechanisms, where blue $(\mathrm{HH}+\mathrm{VV})$ represents surface scattering, green $(2 \mathrm{HV})$ represents volume scattering, and red (HH-VV) represents dihedral scattering or a large copolar difference. The Summit L-band image [Fig. 5(a)] is relatively homogeneous with stronger surface scattering (blue) in near range. P-band Summit [Fig. 5(b)], with deeper penetration depths and less scattering from firn crystals, reveals the presence of multiple ice features, including volume scattering (green) from a well-defined area of possible melt features in 


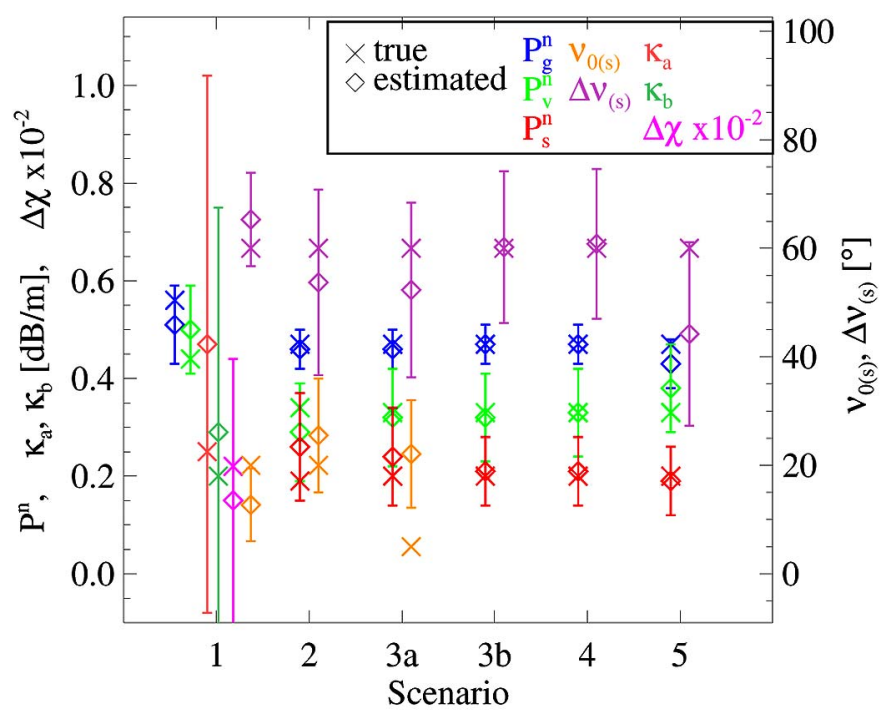

Fig. 4. Simulation results for polarimetric decomposition scenarios without a priori information. True parameter values are shown with a cross (x), and estimated values (including standard deviation error bars) are shown with a diamond $(\diamond)$.

near range and a long ridgelike feature in far range. For the Etonbreen test site, both L- and P-band [Fig. 5(c) and (d)] show similar features, with areas of increased volume scattering and, particularly in near range, areas of increased HH-VV backscatter (red), potentially due to horizontally oriented features.

Fig. 6 shows backscatter coefficients $\left(\sigma^{0}\right)$ averaged through azimuth to reveal trends with incidence angle for the March data; trends in April are similar and are therefore not shown. Decreasing trends in $\sigma^{0}$ with incidence angle are seen for both test sites, particularly for the copolarized channels which are expected to experience significant surface scatter at the snowice interface. Summit backscattering coefficients are approximately $15 \mathrm{~dB}$ larger than those at Etonbreen for L-band and $7 \mathrm{~dB}$ larger for P-band, which could be attributed to increased volume scattering at Summit as well as to smoother ice layers at Etonbreen, thus decreasing the amount of backscattered energy.

As the decomposition approach described in Section III relies on the presence (or lack) of reflection symmetry, correlations in the experimental data are examined. Coherence magnitude histograms of the three linear polarization combinations are shown for the March acquisitions in Fig. 7 (April values are similar and are thus not shown). Copolar coherences are high for both test sites and frequencies, suggesting strong surface scattering. At Summit, cocross correlations are quite low in Fig. 7(a) and (b), with mean values of $\sim 0.25$ or less. At Etonbreen [Fig. 7(c) and (d)], cocross correlations are slightly elevated compared to Summit and have longer tails, implying that, in certain areas, reflection symmetry may not apply. Alternatively, the degree of reflection symmetry can be determined by comparing the determinant of the observed covariance matrix $\operatorname{det}\left(\boldsymbol{C}_{\mathrm{obs}}^{\boldsymbol{H} \boldsymbol{V}}\right)$ with the determinant of the covariance matrix obtained using the five nonzero covariance elements in the reflection symmetric case, as suggested in [40]. Fig. 8 shows the $\operatorname{det}\left(\boldsymbol{C}_{\text {obs }}^{\boldsymbol{H} \boldsymbol{V}}\right) / \operatorname{det}\left(\boldsymbol{C}_{\text {refl sym }}^{\boldsymbol{H} \boldsymbol{V}}\right)$ ratio for the March data; values close to one indicate the presence of reflection symmetry. Again, Summit displays strong reflection symmetry at both

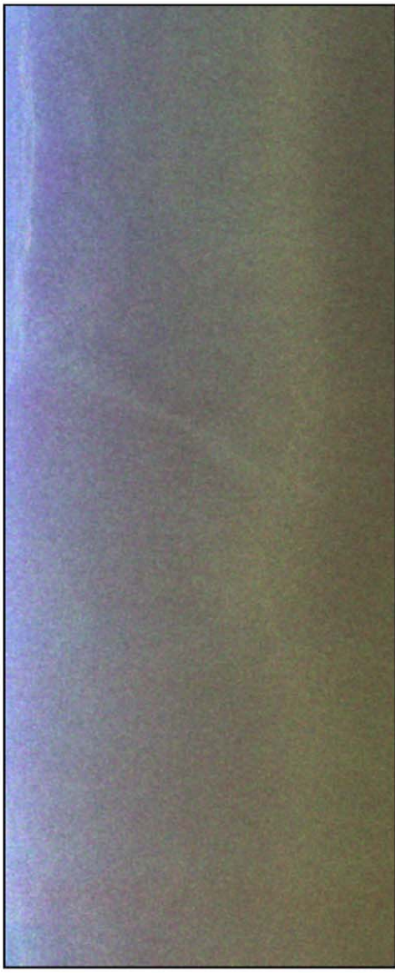

(a) Summit, L-band

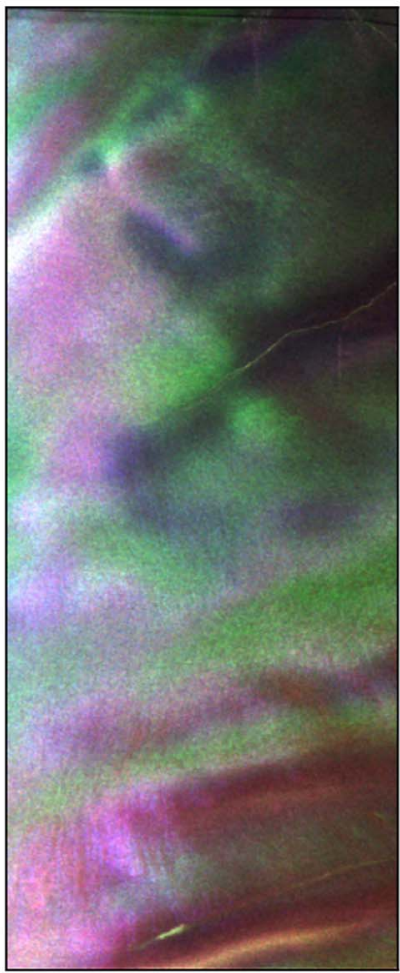

(c) Eton, L-band

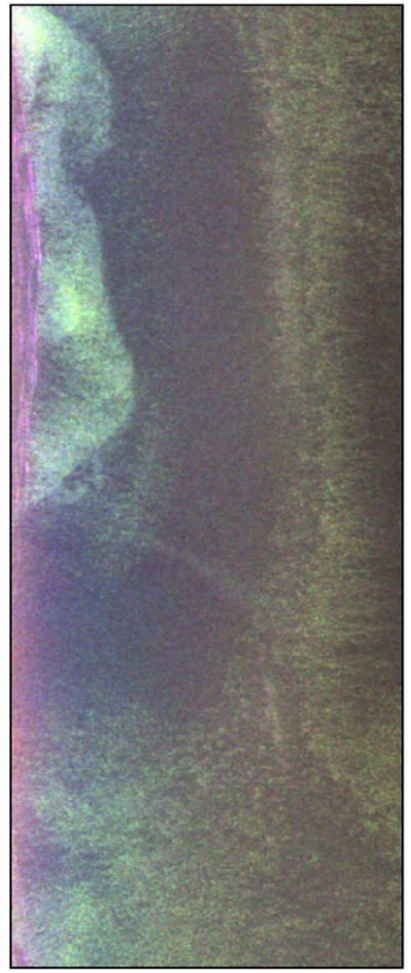

(b) Summit, P-band

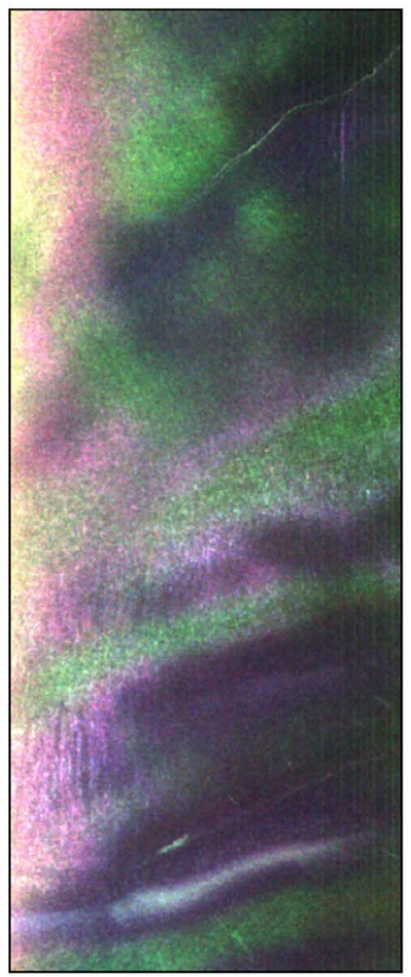

(d) Eton, P-band
Fig. 5. Pauli RGB decomposition of March PolSAR data in slant-range geometry of the Austfonna ice cap. Flight (azimuth) direction is from bottom to top, and range direction is from left to right. L- and P-band images represent approximately the same area. 

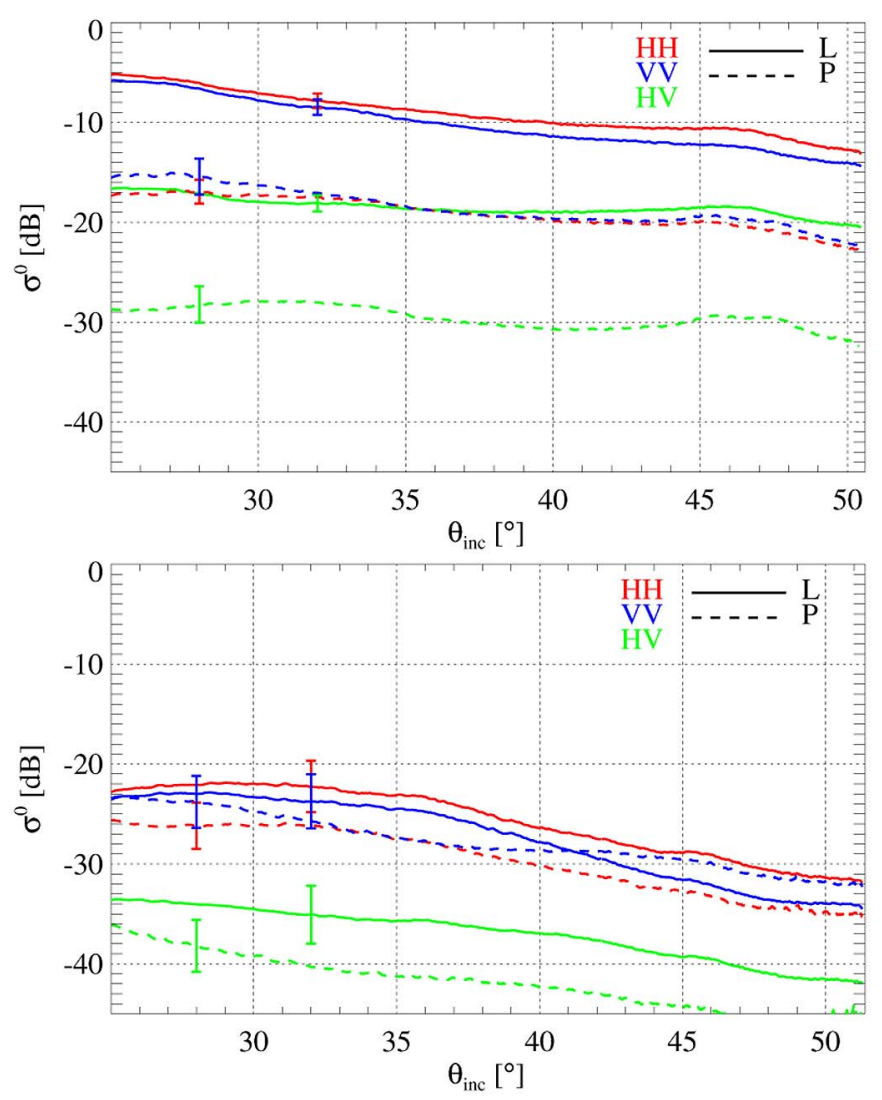

Fig. 6. Backscattering coefficients for (top) Summit and (bottom) Etonbreen versus incidence angle (data have been averaged through azimuth) for the March acquisition. Mean standard deviation (the average of standard deviations taken for each azimuth line) is also plotted.

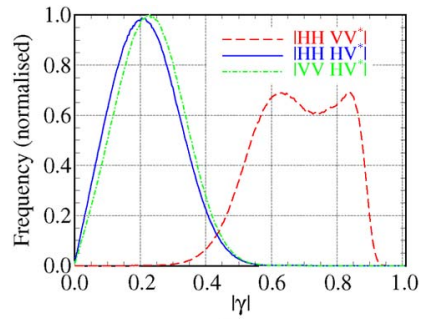

(a)

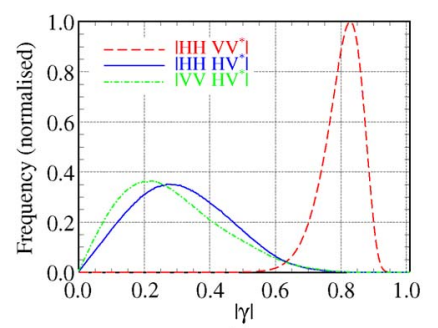

(c)

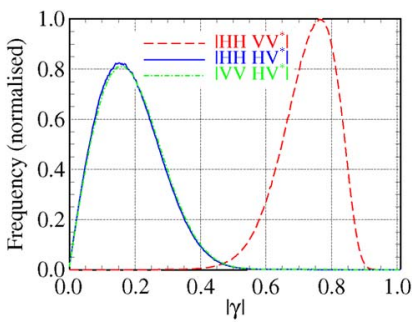

(b)

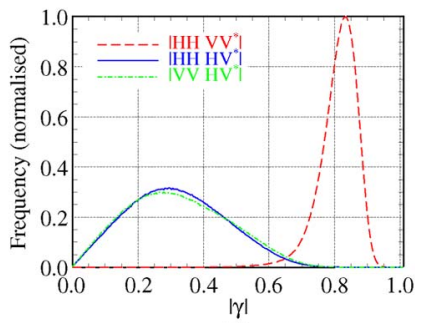

(d)
Fig. 7. Copolar $\left(\mathrm{HH} \mathrm{VV}^{*}\right)$ and cocross $\left(\mathrm{HH} \mathrm{HV}^{*}\right.$ and $\mathrm{VV}_{\mathrm{HV}} \mathrm{HV}^{*}$ ) coherence magnitude histograms for the March acquisitions. (a) Summit, L-band. (b) Summit, P-band. (c) Eton, L-band. (d) Eton, P-band.

frequencies, and Etonbreen is largely reflection symmetric, although there are patches where it does not apply which could indicate areas of scattering from a potential oriented volume or oriented sastrugi (with $\nu_{0} \neq 0^{\circ}, 90^{\circ}$ ). Considered as a whole, however, cocross correlations are fairly low over both test sites (and the reflection symmetry metric is close to one), and inver-

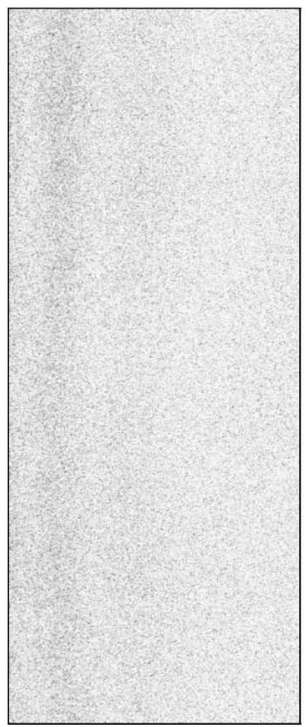

(a)

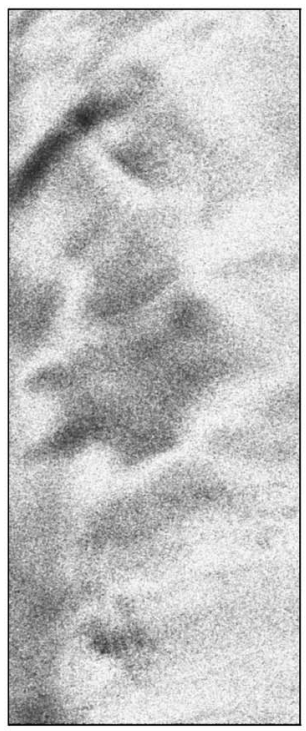

(c)

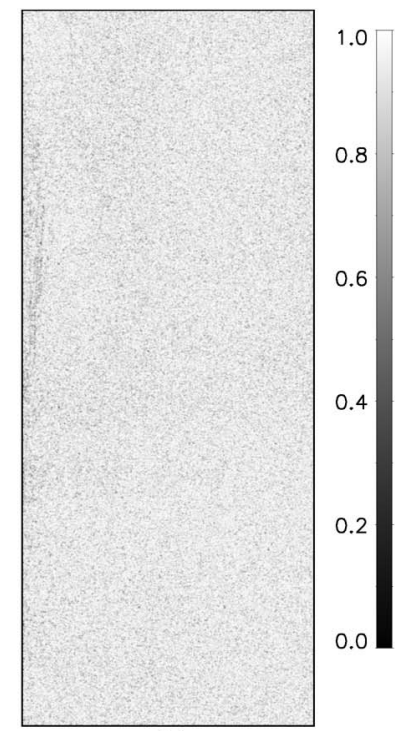

(b)

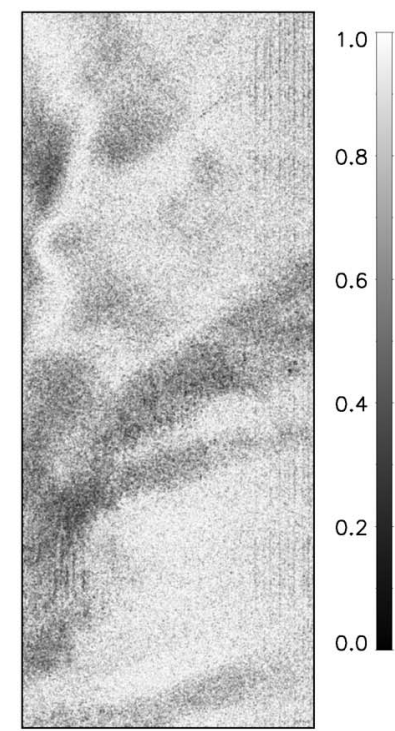

(d)
Fig. 8. Degree of reflection symmetry evaluated using a ratio of covariance matrix determinants [40] for the March acquisitions. A value close to one (white) represents strong reflection symmetry. (a) Summit, L-band. (b) Summit, P-band. (c) Eton, L-band. (d) Eton, P-band.

sion stability is improved under assumption of reflection symmetry even in the presence of small deviations from true symmetry (Section III-B, scenario 3) such that reflection symmetry will be assumed for decomposition purposes in this paper.

The copolarization ratio $|\mathrm{HH}|^{2} /|\mathrm{VV}|^{2}$ is also of interest as it is an indicator of dominant scattering characteristics. In terms of (24), values of $<1$ can indicate SPM surface scattering or the presence of vertically oriented features, $\simeq 1$ is evidence of a rough surface or random volume scattering, and $>1$ is consistent with scattering from horizontally oriented features. It is seen in Fig. 6 that $\sigma_{\mathrm{HH}}^{0}>\sigma_{\mathrm{VV}}^{0}$ for L-band at both test sites, whereas for P-band in March, the copolar $\sigma^{0}$ 's are more similar. Fig. 9 shows the copolar ratios for both March and April acquisitions. The temporal differences are discussed in the subsequent section, but it is seen that in April, the ratio is greater than unity nearly everywhere. This is not an isolated 


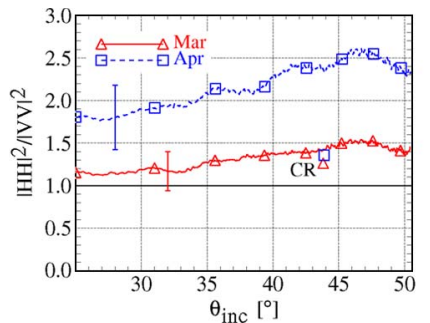

(a)

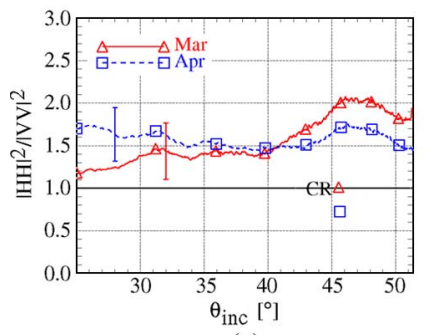

(c)

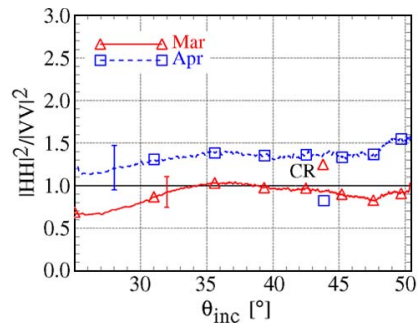

(b)

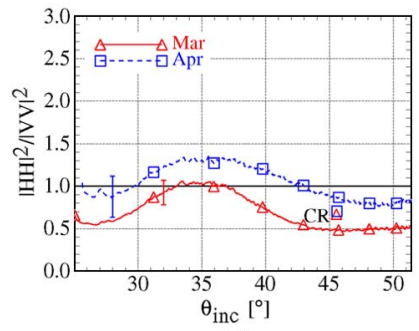

(d)
Fig. 9. Copolarization ratios $|\mathrm{HH}|^{2} /|\mathrm{VV}|^{2}$ versus incidence angle for (triangle, solid line) March and (square, dotted line) April acquisitions. For reference, a solid black line at a ratio of one and observed values at CR are also plotted. (a) Summit, L-band. (b) Summit, P-band. (c) Eton, L-band. (d) Eton, P-band.

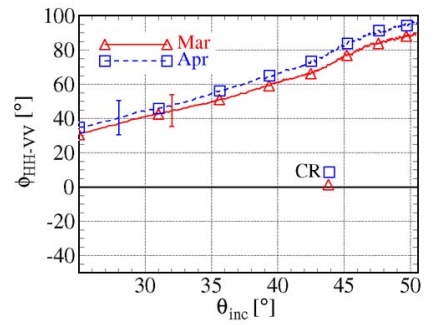

(a)

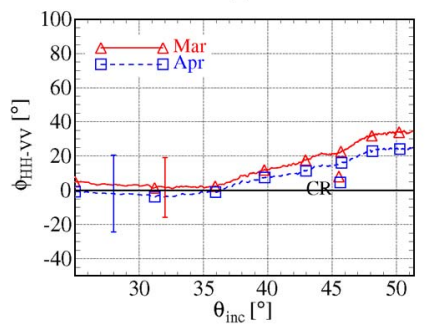

(c)

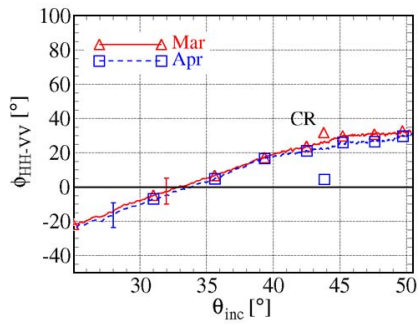

(b)

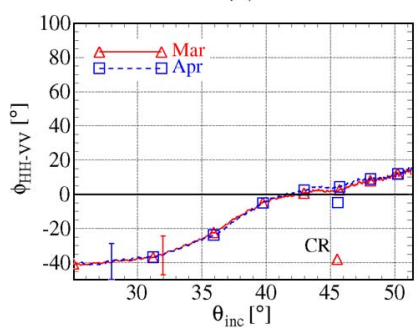

(d)
Fig. 10. Copolarization phase differences $\phi_{\mathrm{HH}-\mathrm{VV}}$ versus incidence angle for (triangle, solid line) March and (square, dotted line) April acquisitions. For reference, a solid black line at a phase difference of zero and observed values at CRs are also plotted. (a) Summit, L-band. (b) Summit, P-band. (c) Eton, L-band. (d) Eton, P-band.

result as larger $\mathrm{HH}$ than VV backscattering coefficients have also been reported for glacier ice at L- and P-band using the AIRSAR sensor of the NASA Jet Propulsion Laboratory over Greenland in [9] and for SIR-C L-band data over certain highelevation areas in Tibet in [13], although it was not attempted to explain the origin of these differences. The combination of high circular polarization ratios $\left(\sigma_{\mathrm{RR}}^{0} / \sigma_{\mathrm{RL}}^{0}>1\right)$ and high linear polarization ratios $\left(\sigma_{\mathrm{HV}}^{0} / \sigma_{\mathrm{HH}}^{0}>1 / 3\right)$ as observed in [8] and [9] was, however, not seen in the Svalbard data used in this work.

A further polarimetric quantity of interest is the copolar phase difference $\phi_{\mathrm{HH}-\mathrm{VV}}$ due to its relatively large values for glacier ice. Fig. 10 shows $\phi_{\mathrm{HH}-\mathrm{VV}}$ for both March and April. The clearly nonzero values suggest the presence of an oriented volume. As shown in (19), for an infinite volume, even a very small $\Delta \chi$ (corresponding to a slight orientation) can lead to a large copolar phase difference. For instance, using the parameters from Table III yields $\phi_{\mathrm{AA}-\mathrm{BB}} \simeq 50^{\circ}$ in the volume component. The phase differences in Fig. 10 show a wide variation between $-40^{\circ}$ and $90^{\circ}$, dependent on both frequency and test site, although an increasing trend in the phase with incidence angle is observed for all passes. An infinite oriented volume alone is not able to explain such large phase variations with incidence angle; (19) has no incidence angle dependence on the copolar phase in the eigenbasis and therefore displays only minimal changes with $\theta_{\text {inc }}$ upon projection to the HV basis. The incidence angle trend is instead attributed to a decreased ground contribution (for which $\phi_{\beta} \simeq 0^{\circ}$ ) with increasing $\theta_{\text {inc }}$, resulting in a larger relative oriented volume contribution and larger phase differences at higher $\theta_{\text {inc }}$.

Only a few copolar phase results are quoted in the literature as most research has been conducted with backscattering power coefficients, but for $\theta_{\mathrm{inc}}>40^{\circ}, \phi_{\mathrm{HH}-\mathrm{VV}}=-29^{\circ}$ and $-1^{\circ}$ were reported for L- and P-band over Greenland [9], while the study in [12] cites values of $18^{\circ}$ (L-band) and $7^{\circ}$ (P-band) in the Alps using the same sensor. Although an accurate modeling of the copolar phase for glacier ice is not yet available and will perhaps require fully phase-coherent models, taking into account multiple scattering and coupling between scattering mechanisms, a combined oriented volume and surface response can explain some of the observed properties.

2) Temporal PolSAR Signatures: Comparisons are made between the two temporal acquisitions to examine the evolution and transformation of SAR ice signatures in the intervening time period of one month. Fig. 9 shows the copolar ratios for March and April, where the ratio for a $1.5-\mathrm{m}$ corner reflector (CR) in each scene is also given to confirm that the differences are not due to a calibration error. Fig. 10 shows the copolar phase differences for both acquisitions. Theoretically, a CR has $|\mathrm{HH}|^{2} /|\mathrm{VV}|^{2}=1$ and copolar phase difference $\phi_{\mathrm{HH}-\mathrm{VV}}=0^{\circ}$, although these values may be biased by the background ice corrupting the CR signal. Particularly large variations are seen in the CR copolar phase differences at P-band in Fig. 10(b) and (d), which are attributed to a low signal-to-clutter ratio, since the $1.5-\mathrm{m} \mathrm{CRs}$ are suboptimal for P-band.

Although the copolar phase differences between acquisitions in Fig. 10 are minimal, the changes in the polarimetric ratios between March and April in Fig. 9 are of interest. No melt events are suspected to have occurred between the two acquisitions as automatic weather stations (AWSs) positioned close to both sites confirmed that temperatures remained below zero. In the absence of melt, it is postulated that changes could only have occurred at the glacier surface, likely due to sastrugi formation. The AWSs recorded a major storm in early April with peak wind speeds exceeding $20 \mathrm{~m} / \mathrm{s}$ and significant precipitation which could have led to large changes in the surface structure. As no significant variations were observed in the copolar phase difference, it is assumed that the underlying volume remained unchanged, whereas the sastrugi component (shown in (24) to influence the backscattered powers) is able to account for changes in the copolar ratio without introducing an additional phase component. 


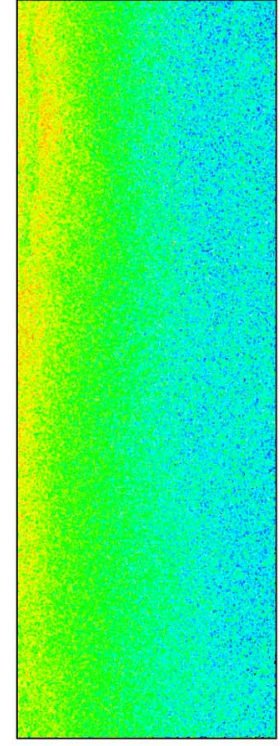

(a) $P_{g}^{n}$ (L-band)

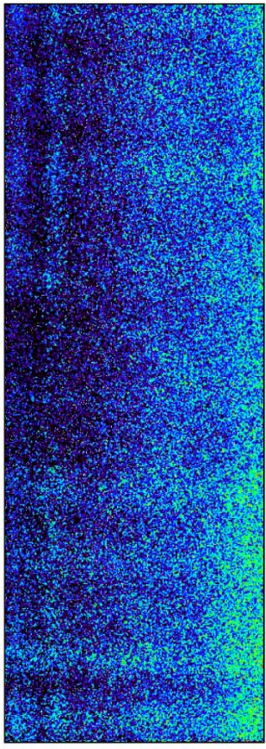

(b) $P_{v}^{n}$ (L-band)

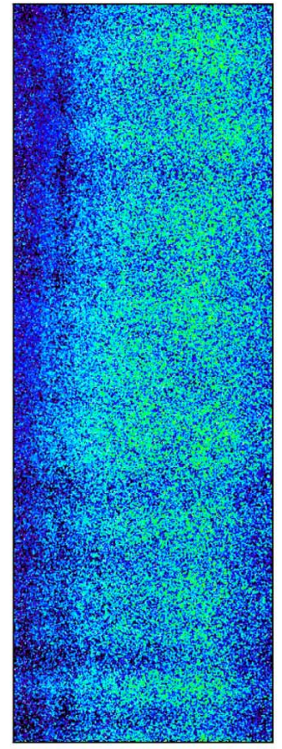

(c) $P_{s}^{n}$ (L-band)

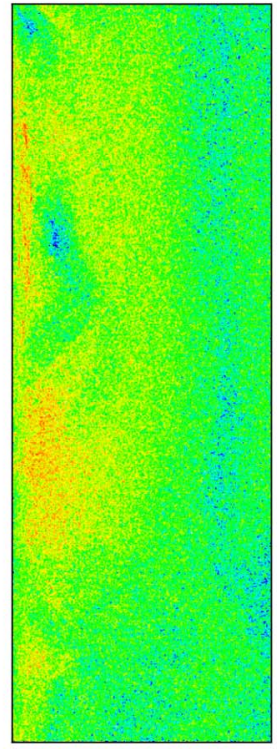

(d) $P_{g}^{n}$ (P-band)

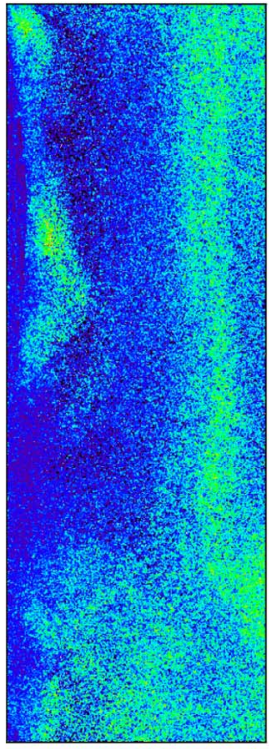

(e) $P_{v}^{n}$ (P-band)

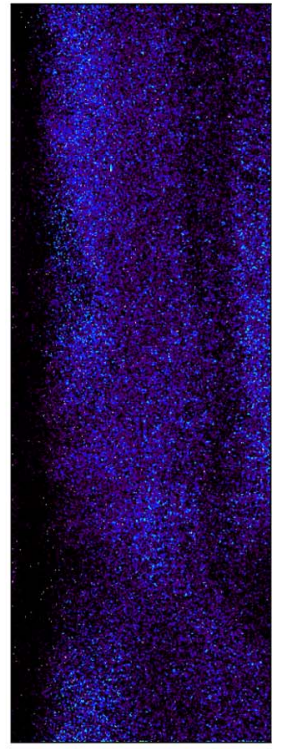

(f) $P_{s}^{n}$ (P-band)

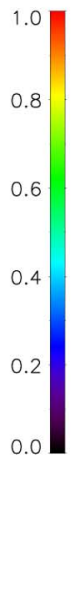

Fig. 11. Polarimetric decomposition of Summit, March PolSAR data for (a)-(c) L-band and (d)-(f) P-band, where $P_{g}^{n}, P_{v}^{n}$, and $P_{s}^{n}$ represent normalized ground, volume, and sastrugi powers, respectively.

\section{Results}

Polarimetric decomposition as described in Section III-A was applied to the experimental data. Based on the polarimetric signatures of the SAR data, it was determined that the sastrugi component is necessary to account for temporal differences in backscatter, and thus, the three-component surface, random volume, and sastrugi decomposition is chosen for inversion. Although the data display potential oriented volume effects, a random volume is used since a combined model with surface, oriented volume, and oriented sastrugi has too many unknowns to invert using a single covariance matrix. It is recognized that this may lead to possible errors in the inversion, the consequences of which were simulated in Section III-B (scenario 5). Decorrelation of the crosspolar channels was used to estimate the noise power in (24) using the method described in [38]. Signal-to-noise ratios were above $25 \mathrm{~dB}$ for all passes except Etonbreen, P-band, for which the additive noise correction becomes necessary.

At both test sites, cocross covariance elements had low magnitudes, and thus, reflection symmetry is assumed. The system of equations is balanced, consisting of four radar observables (three magnitudes $\left|S_{\mathrm{HH}}\right|^{2},\left|S_{\mathrm{VV}}\right|^{2},\left|S_{\mathrm{HV}}\right|^{2}$ and complex value $\left.\left\langle S_{\mathrm{HH}} S_{\mathrm{VV}}^{*}\right\rangle\right)$ and five real unknowns $f_{g}, f_{v}, f_{s}, \phi_{\beta}$, and $\Delta \nu_{s}$, where $\nu_{0} s$ is fixed to zero due to the strong $\mathrm{HH}$ response. $\nu_{0} s$ could have been chosen on a pixel-by-pixel basis depending on the value of $|\mathrm{HH}|^{2} /|\mathrm{VV}|^{2}$, but such small-scale fluctuations of mean sastrugi orientation are inconsistent with reality. However, in the presence of more complex topography, longer and wider scenes from spaceborne sensors, or larger absolute powers of the cocross covariance elements, a different value of $\nu_{0} s$ should be used or estimated from the data.

Illustrations of the normalized power components for L- and P-band March acquisitions at both Summit and Etonbreen are shown in Figs. 11 and 12. Fig. 13 shows inverted sastrugi distribution widths for March L-band. Apart from the relative sastrugi and volume powers, results for April are comparable to those for March and are not displayed. Table I shows the mean normalized power levels and distribution widths averaged over each image as well as inversion rates for both March and April acquisitions. This gives an overview of the relative scattering contributions, although values can be misleading due to inhomogeneities within the scene, particularly at Etonbreen.

\section{DISCUSSION}

\section{A. Decomposition Parameters}

All passes exhibit trends of decreasing surface power with increasing incidence angle as expected from scattering theory. At Summit L-band [Fig. 11(a)-(c)], the scene is very homogeneous, and few ice features are discernible. At P-band [Fig. 11(d)-(f)], more ice structure is visible, with increased volume scatter $P_{v}^{n}$ in an irregularly shaped area in the upper left and along a ridge-type feature in far range [Fig. 11(e)]. Since these features are not visible at L-band, this suggests that these areas are indeed volume-type scatter and have been correctly identified as such by the decomposition. As Summit lies in the firn zone, these regions of volume scattering could correspond to areas of enhanced melt features such as ice pipes and lenses. Relative volume contributions $P_{v}^{n}$ 's were larger for Summit than for Etonbreen at both frequencies. This is expected as Summit is in the firn zone with many high-backscatter ice inclusions, whereas Etonbreen has fewer volume scatterers on the order of the wavelength.

Sastrugi power $P_{s}^{n}$ is greater at L-band than at P-band, perhaps because continuous sastrugi segments are more prevalent at L-band as the wavelength $(0.23 \mathrm{~m})$ is nearly four times smaller than at P-band $(0.86 \mathrm{~m})$ and because P-band 


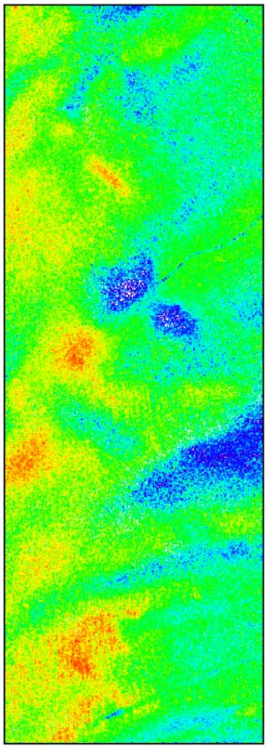

(a) $P_{g}^{n}$ (L-band)

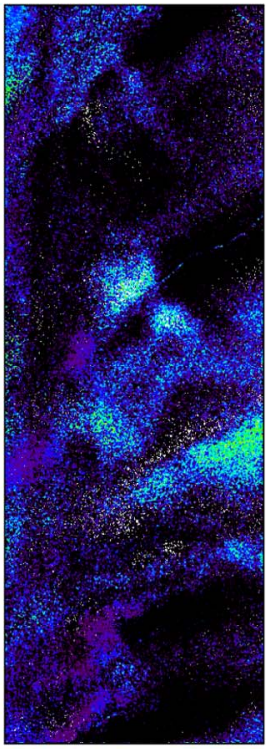

(b) $P_{v}^{n}$ (L-band)

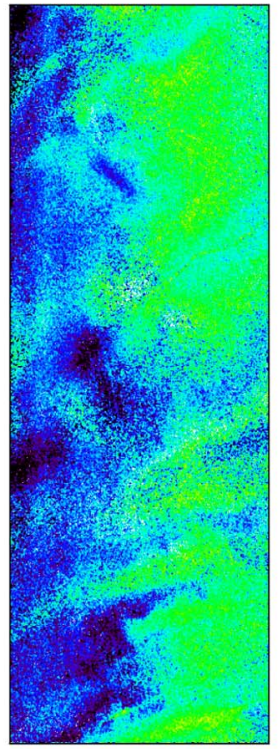

(c) $P_{s}^{n}$ (L-band)

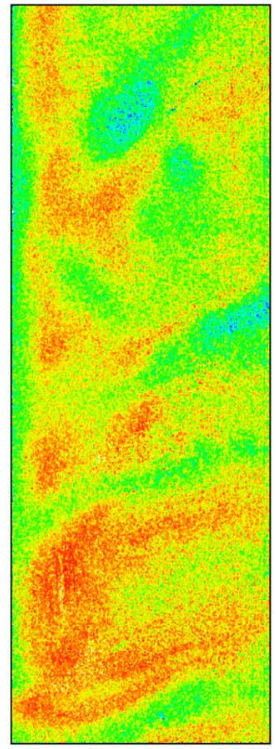

(d) $P_{g}^{n}$ (P-band)

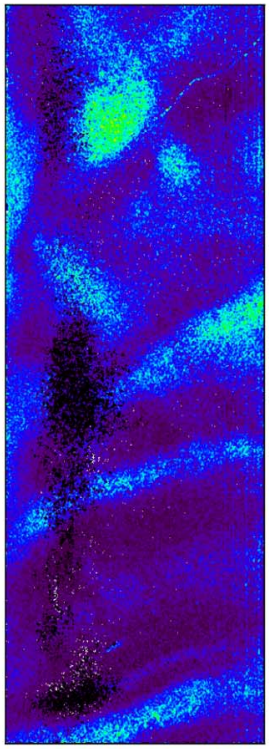

(e) $P_{v}^{n}$ (P-band)

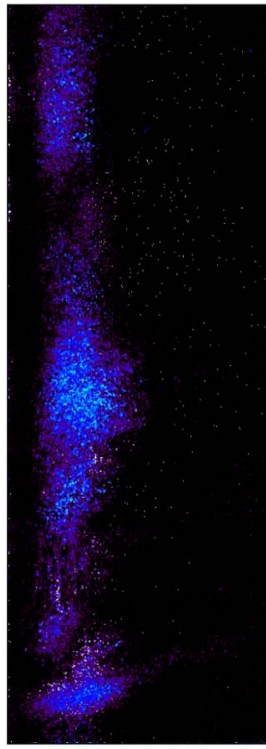

(f) $P_{s}^{n}$ (P-band)

Fig. 12. Polarimetric decomposition of Etonbreen, March PolSAR data for (a)-(c) L-band and (d)-(f) P-band, where $P_{g}^{n}, P_{v}^{n}$, and $P_{s}^{n}$ represent normalized ground, volume, and sastrugi powers, respectively.

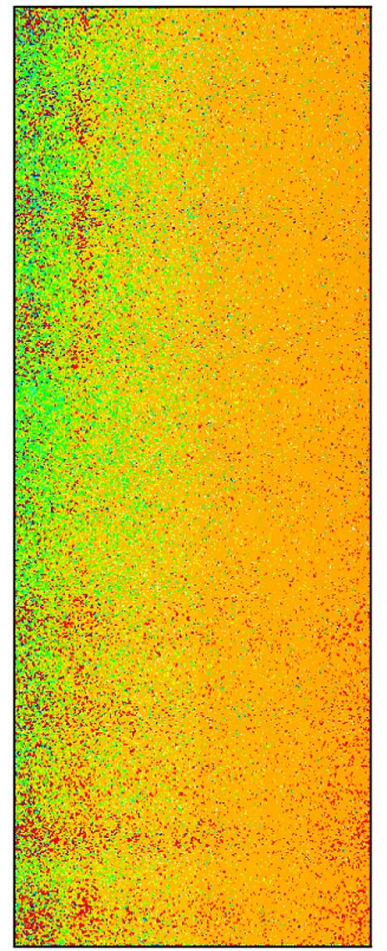

(a) Summit, L-band

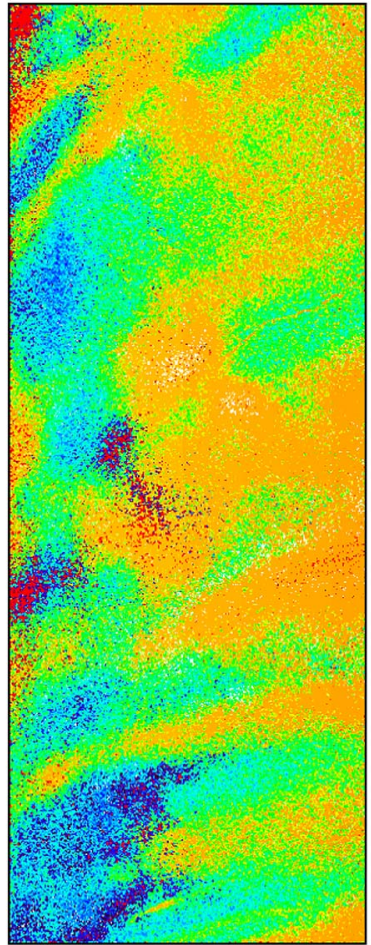

(b) Eton, L-band

Fig. 13. Distribution widths $\Delta \nu_{s}\left[^{\circ}\right]$ from decomposition of L-band March PolSAR data.

penetrates deeper into the ice. Sastrugi distributions widths $\Delta \nu_{s}$ 's (Fig. 13) are fairly wide with means of $\sim 60^{\circ}$ for L-band Summit and Etonbreen. As shown in the simulations of Section III-B (scenario 5), these widths may be significantly underestimated in the presence of an oriented volume. At Etonbreen L-band, sastrugi powers are large in far range [Fig. 12(c)],

TABLE I

Normalized Powers From Three-COMPONENT DeCOMPOSITION OF the Experimental Data Averaged ACross the Entire Image

\begin{tabular}{|c|c|c|c|c|c|c|c|}
\hline & & & $P_{g}^{n}$ & $P_{v}^{n}$ & $P_{s}^{n}$ & $\Delta \nu_{s}$ & $\begin{array}{l}\% \text { non- } \\
\text { invertible }\end{array}$ \\
\hline \multirow[t]{4}{*}{ Summit } & $\mathrm{L}$ & Mar & 0.50 & 0.20 & 0.29 & 64.3 & 1.2 \\
\hline & & Apr & 0.41 & 0.03 & 0.56 & 63.1 & 0.8 \\
\hline & P & Mar & 0.62 & 0.32 & 0.07 & 23.7 & 0.2 \\
\hline & & Apr & 0.61 & 0.14 & 0.25 & 41.9 & 1.4 \\
\hline \multirow[t]{4}{*}{ Eton } & $\mathrm{L}$ & Mar & 0.56 & 0.08 & 0.36 & 54.8 & 1.3 \\
\hline & & Apr & 0.56 & 0.08 & 0.36 & 56.8 & 0.8 \\
\hline & P & Mar & 0.79 & 0.19 & 0.02 & 10.5 & 0.2 \\
\hline & & Apr & 0.74 & 0.15 & 0.10 & 22.3 & 0.5 \\
\hline
\end{tabular}

although these areas also possess large distribution widths [Fig. 13(b)], such that the decomposition could be mistaking a slight orientation in the volume for the presence of sastrugi. In near range, where large (HH-VV) powers were seen in the Pauli image of Fig. 5(c), the orientation distribution widths are narrower and the sastrugi powers are lower, which is more realistic. At P-band, sastrugi powers are very low, such that no meaningful information on the sastrugi widths can be inferred.

\section{B. Temporal Change}

A temporal comparison between March and April shows a clear difference between all passes except Eton L-band (Table I). Both test sites show similar ground responses for March and April, but the higher HH levels present in April are attributed to elevated sastrugi contributions. The relative volume contribution has thereby decreased, perhaps being masked by the overlying sastrugi. The three-component model is thus able to offer a plausible explanation for fluctuations in polarimetric signatures over winter scenes. 


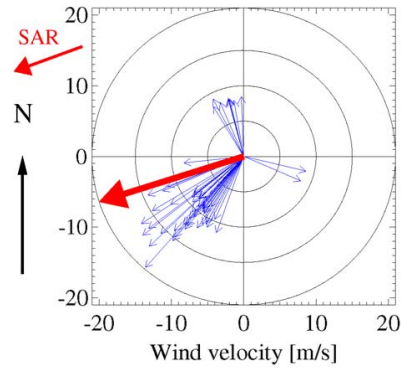

(a)

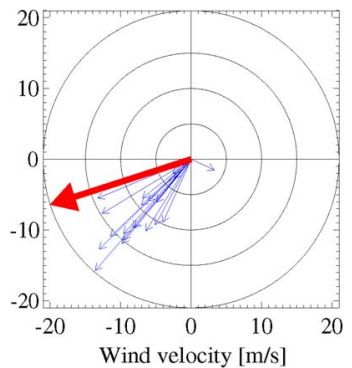

(b)
Fig. 14. Plot of selected wind vectors at an AWS near Etonbreen. Wind intensity is given by the radial distance in meters per second, wind direction is given by the angle from the positive $y$-axis (north). SAR heading is the thick arrow. (a) Intensity of $>8 \mathrm{~m} / \mathrm{s}$. (b) Relative humidity of $>95 \%$.

\section{Validation}

Validation of the decomposition results is made difficult due to a lack of ground measurements. However, some support for the use of $\nu_{0} s=0$ in the decomposition is given by meteorological data. In Fig. 14, the wind intensity and direction recorded hourly during the intervening time period between SAR acquisitions are shown on a polar plot. Vectors are plotted for wind magnitudes of $>8 \mathrm{~m} / \mathrm{s}$ (the minimal threshold for blowing snow [1]) in Fig. 14(a) and for periods with relative humidity of $>95 \%$ (signifying precipitation) in Fig. 14(b), conditions under which sastrugi formation is enhanced. The SAR along-track heading is given in red. Fig. 14(a) and (b) show trends to the southwest which roughly agree with the along-track direction of the SAR. As sastrugi tend to form parallel to the prevailing winds, it is thus expected that sastrugi center orientations lie approximately parallel to the SAR $h$-axis, i.e., aligned along $\nu_{0} \simeq 0$.

It is recommended that ground measurements of sastrugi size, orientation, and extent, as well as snow-ice roughness characteristics, be made simultaneous to SAR acquisitions to aid in the validation of future campaigns.

\section{CONCLUSION}

In this paper, decomposition of PolSAR observables over glacier ice into their constituent scattering mechanisms has been addressed. A model of the polarimetric covariance matrix is used to separate the backscattered signal into surface, volume, and sastrugi components. The novel sastrugi component offers a plausible explanation for changes in the copolar ratio in the absence of melt. Both random and oriented versions of the volumetric scattering model were presented, incorporating polarimetric-dependent transmissivities as well as differential extinction and refractivity effects. The proposed model has the advantage of a direct relation of the angles $\nu$ and $\psi$ to an Earthbased reference system, aiding in physical interpretation. The model has been evaluated and validated on simple forwardmodeling simulations and was applied to L- and P-band polarimetric airborne data over two glacier test sites with varying near-surface structure. Decomposition results conform to known melt characteristics and prevailing winds at the test sites.

The presented decomposition model is not intended and cannot represent the full complexity of the scattering process within glacier ice, but it provides a rationale of the scattering mechanisms involved and is consistent with basic scattering properties such as observed incidence angle trends, polarimetric ratios, and temporal variations in backscatter. The greatest limitation of the decomposition process lies in the model assumptions, the most critical being as follows.

1) Presumption of a random volume which does not account for differential extinction and refractivity. These characteristics are required to reconstruct some of the properties of the observed data, including copolar phases and their behavior with incidence angle.

2) Assumption of reflection symmetry in the decomposition of the experimental data. No precise criteria have been derived to determine when cocross covariance elements become significant.

3) Assumption of a dipole shape for all particles (no variable shape, multishape distributions, shape dependence on depth, etc., are considered).

4) Terrain slope is assumed to be constant over the averaging window, which may not always be applicable to alpine glaciers with steep terrain. In such a scenario, surface range and azimuth slopes must be separated from sastrugi and volume orientations, which is nontrivial.

The assumption of a simplified (random) volume component was necessary because a combined model with surface, oriented volume, and oriented sastrugi has too many unknowns to invert using a single PolSAR image. To distinguish between oriented volume and sastrugi elements, the observation space could be extended using other means such as introducing multiangular acquisitions or interferometric measurements. The use of a multiparameter interferometric approach such as Pol-InSAR offers additional observables to allow a characterization of the vertical distribution of scatterers in ice. This enables parameter estimation with more realistic and less constrained models [41], although the challenge remains in finding a valid model that is consistent with all observables. Further model refinements could also be considered such as a variable particle shape and air-snow transmission coefficients due to the polarizationdependent sastrugi response. Perhaps more complex scattering models must be considered for a dense medium such as ice to account for multiple scattering between particles and coherent scattering between mechanisms, although a lack of ground measurements will continue to hamper such modeling efforts.

Nevertheless, this work is-in our opinion-an important step in separating the highly variable surface and sastrugi components from the underlying volume. Both current (ALOS/PALSAR) and future (e.g., Tandem-L [42] and BIOMASS [43]) satellite missions at L- and P-band benefit from an increased understanding of SAR observables over glacier ice at long wavelengths. Further investigations, including estimation of ice extinctions [44], [45], crystal sizes, and absorption and scattering properties, could be carried out with the isolated volume component. Validation remains challenging due to the lack of in situ measurements, but the increasing availability of fully polarized SAR data over glacial regions has the potential to provide additional insight into their characteristics and their changes over time. 
TABLE II

Model PARAMETERS For THREe-COMPONENT DECOMPOSITION OF GLACIER ICE

\begin{tabular}{ccl}
\hline $\begin{array}{c}\text { Scattering } \\
\text { Mechanism }\end{array}$ & & \multicolumn{1}{c}{ Parameter } \\
\hline \multirow{2}{*}{ Ground } & $f_{g}$ & $\begin{array}{l}\text { power coefficient } \\
\text { ground phase component }\left[{ }^{\circ}\right]\end{array}$ \\
& $\phi_{\beta}$ & \\
Oriented volume & $f_{v}$ & power coefficient \\
& $\nu_{0}$ & mean orientation, $\|$ Earth's surface $\left[^{\circ}\right]$ \\
& $\Delta \nu$ & distribution width of $\nu\left[{ }^{\circ}\right]$ \\
& $\psi_{0}$ & mean orientation, $\perp$ Earth's surface $\left[^{\circ}\right]$ \\
$\Delta \psi$ & distribution width of $\psi\left[{ }^{\circ}\right]$ \\
& $\kappa_{a}$ & extinction along $\vec{a}[\mathrm{~Np} / \mathrm{m}]$ \\
& $\kappa_{b}$ & extinction along $\vec{b}[\mathrm{~Np} / \mathrm{m}]$ \\
& $\Delta \chi$ & differential refractivity $\left(\chi_{a}-\chi_{b}\right)$ \\
& $f_{s}$ & power coefficient \\
& $\nu_{0 s}$ & mean orientation, $\|$ Earth's surface $\left[{ }^{\circ}\right]$ \\
& $\Delta \nu_{s}$ & distribution width of $\nu_{s}\left[{ }^{\circ}\right]$ \\
\hline
\end{tabular}

TABLE III

Simulation PARAMETERS For Polarimetric DeCOMPOSITION SCENARIOS AT L-BAND. FOR THE SPM, $l$ IS THE CORRELATION LENGTH AND $s$ IS THE STANDARD DEVIATION of Vertical SuRface Roughness [23]

\begin{tabular}{|c|c|c|c|c|c|}
\hline \multirow{2}{*}{ Parameter } & \multicolumn{5}{|c|}{ Scenario } \\
\hline & 1 & 2 & 3 & 4 & 5 \\
\hline$\varepsilon_{\text {rsnow }}$ & 1.7 & $"$ & $"$ & $"$ & $"$ \\
\hline$\varepsilon_{r \text { firn }}$ & 2.8 & $"$ & $"$ & $"$ & $"$ \\
\hline$l[\mathrm{~m}]$ & 0.25 & $"$ & $"$ & $"$ & $"$ \\
\hline$s[\mathrm{~m}]$ & 0.01 & $"$ & $"$ & $"$ & $"$ \\
\hline$\phi_{\beta}\left[{ }^{\circ}\right]$ & 0 & $"$ & $"$ & $"$ & $"$ \\
\hline$P_{g}^{n}$ & 0.56 & 0.47 & 0.47 & 0.47 & 0.47 \\
\hline$P_{v}^{g}$ & 0.44 & 0.34 & 0.33 & 0.33 & 0.33 \\
\hline$P_{s}^{n}$ & 0.00 & 0.19 & 0.20 & 0.20 & 0.20 \\
\hline$\psi\left[\left[^{\circ}\right]\right.$ & 0 & & & & 0 \\
\hline$\nu_{0}\left[{ }^{\circ}\right]$ & 20 & & & & 0 \\
\hline$\Delta \nu\left[^{\circ}\right]$ & 60 & & & & 90 \\
\hline$\kappa_{a}[\mathrm{~dB} / \mathrm{m}]$ & 0.25 & & & & 0.25 \\
\hline$\kappa_{b}[\mathrm{~dB} / \mathrm{m}]$ & 0.20 & & & & 0.20 \\
\hline$\Delta \chi\left[\times 10^{-3}\right]$ & 2.2 & & $>$ & $\lambda$ & 2.2 \\
\hline$\nu_{0 s}\left[^{\circ}\right]$ & $\searrow$ & 20 & 5 & 0 & 0 \\
\hline$\Delta \nu_{s}\left[{ }^{\circ}\right]$ & $\backslash$ & 60 & 60 & 60 & 60 \\
\hline
\end{tabular}

\section{APPENDIX}

\section{Simulation PARAMETERS AND RESUltS}

This section presents an overview of the unknown model parameters for the three-component decomposition in Table II as well tables summarizing the simulation parameters (Table III), simulation results (Table IV), and inversion rates (Table V) described in Section III-B.

\section{ACKNOWLEDGMENT}

The authors would like to thank their partners in the ICESAR campaign, including colleagues at the University of Oslo and the Norwegian Polar Institute. The authors would also like to thank M. Davidson for his valuable comments during the
TABLE IV

Simulation Results For POLARIMETRIC DECOMPOSITION SCENARIOS LISTED IN TABLE III. ERRORS $(\Delta)$ ARE COMPUTED AS $\Delta x=\hat{x}-x$, I.E., Estimated Minus TRUe Values

\begin{tabular}{|c|c|c|c|c|c|}
\hline \multirow[b]{2}{*}{ Parameter } & \multirow[b]{2}{*}{ True } & \multicolumn{2}{|c|}{ With a priori } & \multicolumn{2}{|c|}{ Without a priori } \\
\hline & & Estimated & $\Delta$ & Estimated & $\Delta$ \\
\hline \multicolumn{6}{|l|}{ Scenario 1} \\
\hline$P_{q}^{n}$ & 0.56 & $0.55 \pm 0.05$ & -0.01 & $0.51 \pm 0.08$ & -0.05 \\
\hline$P_{v}^{n}$ & 0.44 & $0.46 \pm 0.06$ & -0.02 & $0.50 \pm 0.09$ & +0.06 \\
\hline$\nu_{0}\left[^{\circ}\right]$ & 20.0 & $19.9 \pm 1.5$ & -0.1 & $12.7 \pm 6.7$ & -7.3 \\
\hline$\Delta \nu\left[{ }^{\circ}\right]$ & 60.0 & $59.7 \pm 2.0$ & -0.3 & $65.3 \pm 8.6$ & +5.3 \\
\hline$\kappa_{a}[\mathrm{~dB} / \mathrm{m}]$ & 0.25 & $0.31 \pm 0.13$ & +0.06 & $0.47 \pm 0.55$ & +0.22 \\
\hline$\kappa_{b}[\mathrm{~dB} / \mathrm{m}]$ & 0.20 & $0.23 \pm 0.09$ & +0.03 & $0.29 \pm 0.46$ & +0.09 \\
\hline$\Delta \chi\left[\times 10^{-3}\right]$ & 2.2 & $3.9 \pm 3.2$ & +1.7 & $1.5 \pm 2.9$ & -0.7 \\
\hline \multicolumn{6}{|l|}{ Scenario 2} \\
\hline$P_{q}^{n}$ & 0.47 & $0.46 \pm 0.04$ & -0.01 & $0.46 \pm 0.04$ & -0.01 \\
\hline$P_{v}^{n}$ & 0.34 & $0.28 \pm 0.10$ & -0.06 & $0.29 \pm 0.10$ & -0.05 \\
\hline$P_{s}^{n}$ & 0.19 & $0.28 \pm 0.10$ & +0.09 & $0.26 \pm 0.11$ & -0.07 \\
\hline$\nu_{0 s}\left[{ }^{\circ}\right]$ & 20.0 & $23.9 \pm 7.8$ & +3.9 & $25.5 \pm 10.5$ & +3.5 \\
\hline$\Delta \nu_{s}\left[{ }^{\circ}\right]$ & 60.0 & $57.0 \pm 11.5$ & -3.0 & $53.7 \pm 17.1$ & -6.3 \\
\hline \multicolumn{6}{|l|}{ Scenario 3a } \\
\hline$P_{g}^{n}$ & 0.47 & $0.46 \pm 0.04$ & -0.01 & $0.46 \pm 0.04$ & -0.01 \\
\hline$P_{v}^{n}$ & 0.33 & $0.21 \pm 0.10$ & -0.02 & $0.32 \pm 0.10$ & -0.01 \\
\hline$P_{s}^{n}$ & 0.20 & $0.25 \pm 0.10$ & +0.05 & $0.24 \pm 0.10$ & +0.0 \\
\hline$\nu_{0 s}\left[^{\circ}\right]$ & 5.0 & $21.0 \pm 7.9$ & +16.0 & $22.1 \pm 9.9$ & +17.1 \\
\hline$\Delta \nu_{s}\left[{ }^{\circ}\right]$ & 60.0 & $53.8 \pm 12.5$ & -6.2 & $52.3 \pm 16.1$ & -7.7 \\
\hline \multicolumn{6}{|l|}{ Scenario 3b } \\
\hline$P_{g}^{n}$ & 0.47 & $0.46 \pm 0.04$ & -0.01 & $0.47 \pm 0.04$ & 0.00 \\
\hline$P_{v}^{g}$ & 0.33 & $0.33 \pm 0.09$ & 0.00 & $0.32 \pm 0.09$ & -0.01 \\
\hline$P_{s}^{n}$ & 0.20 & $0.21 \pm 0.08$ & +0.01 & $0.21 \pm 0.07$ & +0.01 \\
\hline$\nu_{0 s}\left[^{\circ}\right]$ & 5.0 & FIX & & FIX & $\backslash$ \\
\hline$\Delta \nu_{s}\left[{ }^{\circ}\right]$ & 60.0 & $58.7 \pm 14.6$ & -1.3 & $60.2 \pm 14.0$ & 0.2 \\
\hline \multicolumn{6}{|l|}{ Scenario 4} \\
\hline$P_{g}^{n}$ & 0.47 & $0.47 \pm 0.04$ & 0.00 & $0.47 \pm 0.04$ & $0.0 c$ \\
\hline$P_{v}^{n}$ & 0.33 & $0.33 \pm 0.09$ & 0.00 & $0.33 \pm 0.09$ & 0.00 \\
\hline$P_{s}^{n}$ & 0.20 & $0.21 \pm 0.08$ & +0.01 & $0.21 \pm 0.07$ & +0.01 \\
\hline$\nu_{0 s}\left[^{\circ}\right]$ & 0.0 & FIX & $\searrow$ & FIX & $\backslash$ \\
\hline$\Delta \nu_{s}\left[{ }^{\circ}\right]$ & 60.0 & $58.6 \pm 14.8$ & -1.4 & $60.8 \pm 13.8$ & +0.8 \\
\hline \multicolumn{6}{|l|}{ Scenario 5} \\
\hline$P_{g}^{n}$ & 0.47 & $0.43 \pm 0.05$ & -0.04 & $0.43 \pm 0.05$ & -0.0 \\
\hline$P_{v}^{n}$ & 0.33 & $0.39 \pm 0.09$ & 0.06 & $0.38 \pm 0.09$ & +0.05 \\
\hline$P_{s}^{n}$ & 0.20 & $0.18 \pm 0.07$ & -0.02 & $0.19 \pm 0.07$ & -0.01 \\
\hline$\nu_{0 s}\left[{ }^{\circ}\right]$ & 0.0 & FIX & & FIX & \\
\hline$\Delta \nu_{s}\left[{ }^{\circ}\right]$ & 60.0 & $42.4 \pm 17.1$ & -17.6 & $44.2 \pm 16.9$ & -15.8 \\
\hline
\end{tabular}

TABLE V

Percentage Noninvertible Pixels FOR EACH DECOMPOSITION SCENARIO

\begin{tabular}{ccc}
\hline \multirow{2}{*}{ Scenario } & \multicolumn{2}{c}{ \% Non-invertible } \\
& w a priori & w/o a priori \\
\hline \multirow{2}{*}{} & & \\
1 & 0.9 & 11.5 \\
2 & 0.3 & 2.1 \\
$3 \mathrm{a}$ & 0.3 & 1.7 \\
$3 \mathrm{~b}$ & 0.7 & 1.0 \\
4 & 0.5 & 1.1 \\
5 & 2.8 & 4.3 \\
\hline
\end{tabular}

campaign and preliminary data analysis and also the reviewers for their constructive comments and recommendations for this paper. 


\section{REFERENCES}

[1] D. H. Bromwich, T. R. Parish, and C. A. Zorman, "The confluence zone of the intense katabatic winds at Terra Nova Bay, Antarctica, as derived from airborne sastrugi surveys and mesoscale numerical modeling," J. Geophys. Res., vol. 96, no. D5, pp. 5495-5509, 1990.

[2] M. Ledroit, F. Rémy, and J.-F. Minster, "Observations of the Antarctic ice sheet with the Seasat scatterometer: Relation to katabatic-wind intensity and direction," J. Glaciol., vol. 39, no. 132, pp. 385-396, 1993.

[3] S. R. Cloude and E. Pottier, "A review of target decomposition theorems in radar polarimetry," IEEE Trans. Geosci. Remote Sens., vol. 34, no. 2, pp. 498-518, Mar. 1996.

[4] A. Freeman and S. L. Durden, "A three-component scattering model for polarimetric SAR data," IEEE Trans. Geosci. Remote Sens., vol. 36, no. 3, pp. 963-973, May 1998.

[5] Y. Yamaguchi, T. Moriyama, M. Ishido, and H. Yamada, "Fourcomponent scattering model for polarimetric SAR image decomposition," IEEE Trans. Geosci. Remote Sens., vol. 43, no. 8, pp. 1699-1706, Aug. 2005.

[6] Y. Yajima, Y. Yamaguchi, R. Sato, H. Yamada, and W.-M. Boerner, "POLSAR image analysis of wetlands using a modified four-component scattering power decomposition," IEEE Trans. Geosci. Remote Sens., vol. 46, no. 6, pp. 1667-1673, Jun. 2008.

[7] I. Hajnsek, T. Jagdhuber, H. Schon, and K. P. Papathanassiou, "Potential of estimating soil moisture under vegetation cover by means of PolSAR," IEEE Trans. Geosci. Remote Sens., vol. 47, no. 2, pp. 442-454, Feb. 2009.

[8] E. J. Rignot, S. Ostro, J. van Zyl, and K. C. Jezek, "Unusual radar echoes from the Greenland ice sheet," Science, vol. 261, no. 5129, pp. 1710-1713, Sep. 1993.

[9] E. J. Rignot, "Backscatter model for the unusual radar echoes from the Greenland ice sheet," J. Geophys. Res., vol. 100, no. E5, pp. 9389-9400, 1995.

[10] D. Floricioiu and H. Rott, "Seasonal and short-term variability of multifrequency, polarimetric radar backscatter of alpine terrain from SIR-C/XSAR and AIRSAR data," IEEE Trans. Geosci. Remote Sens., vol. 39, no. 12, pp. 2634-2648, Dec. 2001.

[11] J. Shi, J. Dozier, and H. Rott, "Snow mapping in alpine regions with synthetic aperture radar," IEEE Trans. Geosci. Remote Sens., vol. 32, no. 1, pp. 152-158, Jan. 1994.

[12] H. Rott and R. Davis, "Multifrequency and polarization SAR observations on alpine glaciers," Ann. Glaciol., vol. 17, pp. 98-104, 1993.

[13] A. F. Haldemann and D. O. Muhleman, "Circular-polarization radar properties of high-altitude ice: Western Kunlun Shan and central Andes," J. Geophys. Res., vol. 104, no. E10, pp. 24075-24094, Oct. 1999.

[14] E. W. Hoen and H. Zebker, "Penetration depths inferred from interferometric volume decorrelation observed over the Greenland ice sheet," IEEE Trans. Geosci. Remote Sens., vol. 38, no. 6, pp. 2572-2583, Nov. 2000.

[15] S. Oveisgharan and H. A. Zebker, "Estimating snow accumulation from InSAR correlation observations," IEEE Trans. Geosci. Remote Sens., vol. 45, no. 1, pp. 10-20, Jan. 2007.

[16] J. Dall, K. P. Papathanassiou, and H. Skriver, "Polarimetric SAR interferometry appied to land ice: Modeling," in Proc. EUSAR, Ulm, Germany, May 2004, pp. 247-250.

[17] J. J. Sharma, I. Hajnsek, and K. P. Papathanassiou, "Multi-frequency PolInSAR signatures of a subpolar glacier," in Proc. 3rd Int. Workshop Sci. Appl. SAR Polarimetry Polarimetric Interferometry (PolInSAR), Frascati, Italy, Jan. 22-26, 2007.

[18] G. Sinclair, "The transmission and reception of elliptically polarized waves," Proc. IRE, vol. 38, no. 2, pp. 148-151, Feb. 1950.

[19] A. B. Kostinski and W. Boerner, "On foundations of radar polarimetry," IEEE Trans. Antennas Propag., vol. AP-34, no. 12, pp. 1395-1404, Dec. 1986.

[20] W.-M. Boerner, H. Mott, E. Lüneberg, C. Livingstone, B. Brisco, R. J. Brown, and J. S. Paterson, "Polarimetry in radar remote sensing: Basic and applied concepts," in Principles and Applications of Imaging Radar, Manual of Remote Sensing, 3rd ed. Hoboken, NJ: Wiley, 1998, ch. 5, pp. 271-357.

[21] S. Cloude, Polarisation: Applications in Remote Sensing. London, U.K.: Oxford Univ. Press, 2010.

[22] J.-S. Lee and E. Pottier, Polarimetric Radar Imaging: From Basics to Applications. Boca Raton, FL: CRC Press, 2009.

[23] F. Ulaby, R. Moore, and A. Fung, Microwave Remote Sensing, Active and Passive, vol. 2, Radar Remote Sensing and Surface
Scattering and Emission Theory. Norwood, MA: Addison-Wesley, 1982.

[24] E. W. Hoen, "A correlation-based approach to modeling interferometric radar observations of the Greenland ice sheet," Ph.D. dissertation, Stanford Univ., Stanford, CA, Mar. 2001.

[25] S. R. Cloude, J. Fortuny, J. Lopez-Sanchez, and A. Sieber, "Wideband polarimetric radar inversion studies for vegetation layers," IEEE Trans. Geosci. Remote Sens., vol. 37, no. 5, pp. 2430-2441, Sep. 1999.

[26] M. Neumann, L. Ferro-Famil, and A. Reigber, "Estimation of forest structure, ground, and canopy layer characteristics from multibaseline polarimetric interferometric SAR data," IEEE Trans. Geosci. Remote Sens., vol. 48, no. 3, pp. 1086-1104, Mar. 2010.

[27] M. Neumann, "Remote sensing of vegetation using multi-baseline polarimetric SAR inteferometry: Theoretical modeling and physical parameter retrieval," Ph.D. dissertation, Univ. de Rennes I, Rennes, France, 2009.

[28] V. Lytle and K. Jezek, "Dielectric permittivity and scattering measurements of Greenland firn at 26.5-40 GHz," IEEE Trans. Geosci. Remote Sens., vol. 32, no. 2, pp. 290-295, Mar. 1994.

[29] S. Fujita, T. Matsuoka, T. Ishida, K. Matsuoka, and S. Mae, "A summary of the complex dielectric permittivity of ice in the megahertz range and its applications for radar sounding of polar ice sheets," in Physics of Ice Core Records. Sapporo, Japan: Hokkaido Univ. Press, 2000, pp. $185-212$.

[30] S. Fujita and S. Mae, "Relation between ice sheet internal radio-echo reflections and ice fabric at Mizuho Station," Ann. Glaciol., vol. 17, pp. 269-275, 1993.

[31] T. Matsuoka, S. Fujita, S. Morishima, and S. Mae, "Precise measurement of dielectric anisotropy in ice at $39 \mathrm{GHz}$," J. Appl. Phys., vol. 81, no. 5, pp. 2344-2348, Mar. 1997.

[32] K. Matsuoka, L. Wilen, S. Hurley, and C. Raymond, "Effects of birefringence within ice sheets on obliquely propagating radio waves," IEEE Trans. Geosci. Remote Sens., vol. 47, no. 5, pp. 1429-1443, May 2009.

[33] R. N. Treuhaft and S. R. Cloude, "The structure of oriented vegetation from polarimetric interferometry," IEEE Trans. Geosci. Remote Sens., vol. 37, no. 5, pp. 2620-2624, Sep. 1999.

[34] J.-S. Lee, D. L. Schuler, and T. L. Ainsworth, "Polarimetric SAR data compensation for terrain azimuth slope variation," IEEE Trans. Geosci. Remote Sens., vol. 38, no. 5, pp. 2153-2163, Sep. 2000.

[35] C. Mätzler, "Applications of the interaction of microwaves with natural snow cover," Remote Sens. Rev., vol. 2, no. 2, pp. 259-387, 1987.

[36] F. Ulaby, R. Moore, and A. Fung, Microwave Remote Sensing, Active and Passive, vol. 1, Fundamentals and Radiometry. Norwood, MA: Addison-Wesley, 1981.

[37] R. Bindschadler, "Tracking subpixel-scale sastrugi with Advanced Land Imager," IEEE Trans. Geosci. Remote Sens., vol. 41, no. 6, pp. 1373-1377, Jun. 2003.

[38] I. Hajnsek, E. Pottier, and S. R. Cloude, "Inversion of surface parameters from polarimetric SAR," IEEE Trans. Geosci. Remote Sens., vol. 41, no. 4, pp. 727-743, Apr. 2003.

[39] S. Nghiem, S. Yueh, R. Kwok, and F. Li, "Symmetry properties in polarimetric remote sensing," Radio Sci., vol. 27, no. 5, pp. 693-711, Sep./Oct. 1992.

[40] L. Ferro-Famil and M. Neumann, "Recent advances in the derivation of POL-inSAR statistics: Study and applications," in Proc. EUSAR, Friedrichshafen, Germany, Jun. 2-5, 2008.

[41] R. N. Treuhaft and P. R. Siqueira, "Vertical structure of vegetated land surfaces from interferometric and polarimetric radar," Radio Sci., vol. 35, no. 1, pp. 141-177, Jan./Feb. 2000.

[42] A. Moreira, G. Krieger, I. Hajnsek, K. Papathanassiou, M. Eineder, F. De Zan, M. Younis, and M. Werner, "Tandem-L: Monitoring the Earth's dynamics with InSAR and Pol-InSAR," in Proc. 4th Int. Workshop Sci. Appl. SAR Polarimetry Polarimetric Interferometry (PolInSAR), Frascati, Italy, Jan. 26-30, 2009.

[43] M. Davidson, A. Thompson, and C.-C. Lin, Report for assessment: BIOMASS, ESA SP-1313/2 Candidate Earth Explorer Core Mission, Tech. Rep., Nov. 2008. [Online]. Available: http://www.congrex.nl/09c01/

[44] J. J. Sharma, I. Hajnsek, and K. P. Papathanassiou, "Extinction estimation over glacier ice with long-wavelength Pol-InSAR," IEEE Trans. Geosci. Remote Sens., 2010, submitted for publication.

[45] J. J. Sharma, "Estimation of glacier ice extinction using longwavelength polarimetric interferometric synthetic aperture radar," Ph.D. dissertation, Karlsruher Inst. Technol. (KIT), Karlsruhe, Germany, 2010. 


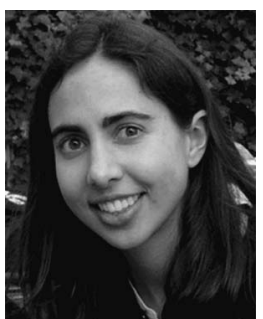

Jayanti J. Sharma (S'05) received the B.Sc. (with distinction) and M.Sc. degrees in geomatics engineering from the University of Calgary, Calgary, AB, Canada, in 2002 and 2005, respectively. She is currently working toward the Ph.D. degree in electrical engineering at the University of Karlsruhe, Karlsruhe, Germany, in cooperation with the Microwaves and Radar Institute, German Aerospace Center (DLR), Wessling, Germany.

From 2003 to 2004, she was with the Radar Systems Group, Defence R\&D Canada-Ottawa. Her research interests include radar polarimetry, polarimetric SAR interferometry, geophysical parameter inversion, and remote sensing of the cryosphere.

Ms. Sharma received the Best Student Paper Award of the European SAR Conference (EUSAR) in 2010.

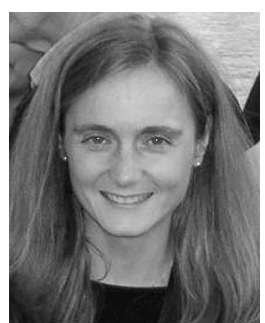

Irena Hajnsek (A'01-M'06-SM'09) received the Dipl. degree (with honors) from the Free University of Berlin, Berlin, Germany, in 1996 and the Dr. degree (with honors) from the Friedrich Schiller University of Jena, Jena, Germany, in 2001.

From 1996 to 1999, she was with the Microwaves and Radar Institute (HR), German Aerospace Center (DLR), Wessling, Germany. From 1999 to 2000, she spent ten months with the Institut d'Electronique et de Télécommunications de Rennes, University of Rennes 1, France, and four months with Applied Electromagnetics (AEL), St. Andrews, U.K., in the frame of the EC-TMR Radar Polarimetry Network. In 2005, for six weeks, she was a Guest Scientist with the University of Adelaide, Adelaide, Australia. Since November 2009, she has been a Professor of Earth observation with the Institute of Environmental Engineering, ETH Zürich, Zurich, Switzerland, and, at the same time, the Head of the Polarimetric SAR Interferometry research group at DLR-HR. Her main research interests are in electromagnetic propagation and scattering theory, radar polarimetry, SAR and interferometric SAR data processing techniques, and environmental parameter modeling and estimation. She is the Science Coordinator of the German satellite mission TanDEM-X.

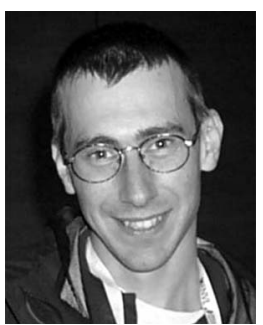

Konstantinos P. Papathanassiou (M'02-SM'03) received the Dipl. Ing. degree (with honors) and the Dr. degree (with honors) from the Technical University of Graz, Graz, Austria, in 1994 and 1999, respectively.

From 1992 to 1994, he was with the Institute for Digital Image Processing (DIBAG) of Joanneum Research, Graz. Between 1995 and 1999, he was with the Microwaves and Radar Institute (HR), German Aerospace Center (DLR), Wessling, Germany. From 1999 to 2000, he was an EU Postdoctoral Fellow with Applied Electromagnetics (AEL), St. Andrews, U.K. Since October 2000, he has been with DLR-HR, where he is currently a Senior Scientist leading the Information Retrieval research group. His main research interests are in polarimetric and interferometric processing and calibration techniques, polarimetric SAR interferometry, and the quantitative parameter estimation from SAR data, as well as in SAR mission design and SAR mission performance analysis.

Dr. Papathanassiou received the IEEE GRSS IGARSS Symposium Prize Paper Award in 1998, the Best Paper Award of the European SAR Conference (EUSAR) in 2002, and the DLR science award in 2002.

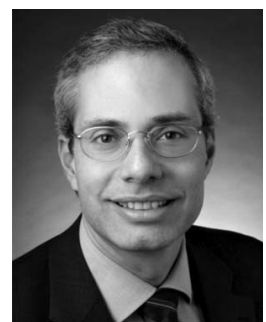

Alberto Moreira (M'92-S'96-F'04) received the B.S.E.E. and M.S.E.E. degrees from the Aeronautical Technological Institute (ITA), São José dos Campos, Brazil, in 1984 and 1986, respectively, and the Eng. Dr. degree (with honors) from the Technical University of Munich, München, Germany, in 1993.

From 1996 to 2001, he was the Chief Scientist and Engineer with the SAR Technology Department, German Aerospace Center (DLR), Wessling, Germany, where since 2001, he has been the Director of the Microwaves and Radar Institute. The institute contributes to several scientific programs and space projects for actual and future airborne and spaceborne SAR missions like TerraSAR-X, TanDEM-X, and Sentinel-1. Since 2003, he has been a Full Professor with the University of Karlsruhe, Karlsruhe, Germany, in the field of microwave remote sensing. In 2006, the mission proposal TanDEM-X led by his institute was approved for the realization phase. He is the initiator and Principal Investigator for this mission. Under his leadership, the DLR airborne SAR system has been upgraded to operate in innovative imaging modes like polarimetric SAR interferometry and SAR tomography. He has more than 280 publications in international conferences and journals. He is the holder of 15 patents in the radar and antenna field. His professional interests and research areas encompass radar end-toend system design and analysis, innovative microwave techniques and system concepts, signal processing, and remote sensing applications.

Prof. Moreira is a member of the IEEE Geoscience and Remote Sensing Society (GRS-S) Administrative Committee (1999-2001, 2004-2009, and 2010 as President), was the Founder and Chair of the German Chapter of the GRS-S (2003-2008), was an Associate Editor for the IEEE GEOSCIENCE AND Remote Sensing LetTers (2003-2007), and has been serving as an Associate and Guest Editor for the IEEE TRANSACTIONS ON GEOSCIENCE AND REMOTE SENSING since 2005. From 2003 to 2008, he served as a member of the Board of Directors of the Information Technology Society of the German Association for Electrical, Electronic and Information Technologies. He is currently the Chair of the Scientific and Technical Council of DLR (2009-2011). He has contributed to the successful series of the European SAR conferences (EUSAR) since 1996 as a member of the Technical Program Committee, the Technical Chairman (2000), the Awards Chairman (2002-2004), the General Chairman (2006), and the Cochairman (2008). In 1995, he received the DLR Science Award. He and his colleagues received the GRS-S Transactions Prize Paper Awards in 1997, 2001, and 2007. He also received the IEEE Nathanson Award for the Young Radar Engineer of the Year in 1999 and the IEEE Kiyo Tomiyasu Field Award in 2007. 